\title{
Photonic Microwave and RF Spectrum Channelizers based on Optical Kerr Micro-combs
}

\author{
M. $\operatorname{Tan}^{1}$, X. Xu' ${ }^{2}$ and D.J. Moss ${ }^{1}$ \\ ${ }^{1}$ Optical Sciences Centre, Swinburne University of Technology, Hawthorn, VIC 3122, Australia \\ ${ }^{2}$ Dept. of Electrical and Computer Systems Engineering, Monash University, Clayton, 3800 VIC, Australia \\ ${ }^{\dagger}$ Corresponding e-mail: dmoss@swin.edu.au
}

\begin{abstract}
We review recent work on broadband RF channelizers based on integrated optical frequency Kerr microcombs combined with passive micro-ring resonator filters, with microcombs having channel spacings of $200 \mathrm{GHz}$ and $49 \mathrm{GHz}$. This approach to realizing RF channelizers offers reduced complexity, size, and potential cost for a wide range of applications to microwave signal detection.
\end{abstract}

Keywords -Microwave photonic, signal channelization, integrated optical frequency comb.

\section{INTRODUCTION}

Nonlinear optics as a means to achieve ultrafast all-optical signal processing has been extremely powerful, and has focused on photonic integrated circuit platforms based on highly nonlinear materials such as silicon [1-3]. The range of signal processing operations that can be performed with nonlinear optical techniques is very large, and includes optical logic [4], optical temporal demultiplexing at ultra-high bit rates from 160Gigabits/s [5] to well over a terabit per second [6], optical performance monitoring based on slow light [7,8], signal regeneration [9,10], and many other functions [1116]. Complementary metal oxide semiconductor (CMOS) compatible platforms are extremely important since they can exploit the extensive global infrastructure established to fabricate computer chips. Since all CMOS compatible material platforms are centrosymmetric, the $2^{\text {rd }}$ order nonlinear response is zero and so nonlinear devices in these platforms have been based on the $3^{\text {rd }}$ order nonlinear susceptibility that accounts for processes including optical third harmonic generation $[11,17-21]$ as well as the instantaneous intensity dependent refractive index termed the Kerr nonlinearity $\left(n_{2}\right)[1,2]$. The efficiency of all-optical devices based on the Kerr nonlinearity depends on the waveguide nonlinear parameter $(\gamma)$, and although silicon waveguides and nanowires have achieved extremely high nonlinear parameters $(\gamma)$, they also display quite high nonlinear optical loss arising from indirect two-photon absorption (TPA) in the telecommunications wavelength region (near $1550 \mathrm{~nm}$ ), since the indirect bandgap of silicon is only $1.17 \mathrm{eV}$ which is much less than twice the photon energy at $1550 \mathrm{~nm}$. Moreover, worse than the intrinsic TPA is the effect of the resulting generated free carriers [2] since the carrier recombination time in silicon can be very long (microseconds), enabling significant carrier densities (and hence loss) to build up. Even if the effect of these carriers can be minimized through the use of $\mathrm{p}-\mathrm{i}-\mathrm{n}$ junctions to sweep out the carriers and greatly reduce the effective carrier lifetime, the intrinsic material nonlinear figure of merit (FOM $=n_{2} /(\beta \lambda)$, where $\beta$ is the TPA and $\lambda$ the wavelength) of silicon is only 0.3 in the telecom band. This is too low to achieve high all-optical performance and is a fundamental property of silicon's bandstructure and therefore cannot be improved. While TPA can be turned around and actually used as a positive tool for some nonlinear functions [22-24], for most processes silicon's low FOM in the telecom band poses a significant limitation. This has been the motivation for research into alternative nonlinear optical platforms, such as chalcogenide glass [25-34], for example. However, although many of these platforms offer significant advantages, most of them are not CMOS compatible, which ultimately is a fundamental consideration to achieve widespread and efficient manufacturing.

New CMOS compatible platforms for nonlinear optics were introduced in 2007/8 that exhibit negligible TPA in the $1550 \mathrm{~nm}$ wavelength regime. These include silicon nitride $[35,36]$ and high-index doped silica glass, trade-named Hydex [37-47]. In addition to having negligible nonlinear absorption even up to many Gigawatts of power, these platforms have a moderate Kerr nonlinearity, resulting in an extremely high nonlinear FOM with a nonlinear parameter $(\gamma)$ that is high enough to realize significant parametric gain. Following the first report of a micro-resonator based optical frequency comb source driven by the Kerr nonlinearity in 2007 [48], the first fully integrated optical parametric oscillators based on microring resonators (MRRs) were reported in $2010[36,37]$ in these new CMOS compatible platforms of SiN and Hydex. Since 2010 the field of integrated micro-combs, or Kerr combs has become one of the largest and most successful fields in optics 
and photonics [47]. They are a new and powerful tool to accomplish many new functions on an integrated chip, due to their very high coherence, while also offering flexible control of their wavelength spacing. Optical micro-combs are produced via optical parametric oscillation driven by modulational instability, or parametric, gain in integrated ring resonators. This offers huge advantages over more conventional approaches including discrete multiple laser wavelength sources. Many breakthroughs have been reported with Kerr micro-combs, from innovative mode-locked lasers [49-52] to quantum optical photonic chips [53-61], ultrahigh bandwidth optical fiber data transmission [62-64], optical neural networks [65-67], integrated optical frequency synthesizers [68] and more, and have been extensively reviewed [47, 69 76]. The success of these new CMOS platforms has motivated the search for even higher performing CMOS compatible platforms including as amorphous silicon [77] and silicon rich silicon nitride [78], searching for the ideal combination of low linear and nonlinear loss together with a high nonlinearity.

A significant application for microcombs has been for signal processing functions for telecommunications and $\mathrm{RF} /$ microwave systems, including RF photonic applications to signal generation and processing for radar systems [79128]. RF photonics is attractive because of its ultra-high bandwidth as well as low transmission loss and strong immunity to electromagnetic interference. There are different RF photonic approaches including methods that map the optical filter response onto the RF domain including devices based on stimulated Brillouin scattering [88-95], that have achieved high $\mathrm{RF}$ resolution - down to $32 \mathrm{MHz}$, a stopband discrimination > $55 \mathrm{~dB}$. A different but equally key approach to reconfigurable RF transfer functions for signal processing has been transversal filter methods [96-100]. These operate by generating progressively delayed and weighted replicas of an RF signal multicast onto many optical carriers, which are subsequently summed via photo-detection. Transverse filters can achieve a wide range of RF functions solely by varying the tap weights, and so this approach is very attractive for advanced dynamically adaptive RF filters. Discrete diode laser arrays [101] and fibre and integrated Bragg grating arrays and sampled gratings [103] have been used to generate the required taps. However, while offering advantages, these methods have increased complexity and footprint, limiting performance due to the limited number of wavelengths. Alternative methods such as electro-optic (EO) or acousto-optic (AO) combs [102,104,105], can help overcome this, but they require high bandwidth modulators and high-frequency RF sources.

Kerr micro-combs [36, 37] have been very successful in their applications to RF systems, providing advantages over other methods of supplying multiple wavelengths for RF photonic systems. They have achieved extremely high bandwidth data communications as well as a wide range of microwave signal processing devices [107-128]. Their comb spacings can be much wider than electro-optic combs, and in many ways EO and micro combs are complementary. EO combs excel at finer spacings from 10's of megahertz to $10-20 \mathrm{GHz}$, while integrated micro-combs typically have much wider spacings from 10's of GHz to 100's of GHz and even THz. Larger comb spacings have much wider Nyquist zones for large RF bandwidth operation, whereas smaller spacings provide many more wavelengths or RF "taps", although at the expense of a smaller Nyquist zones. Micro-combs provide more wavelengths while still having a large FSR, all in a small footprint. For RF transversal filters the number of wavelengths determines the number of channels for RF true time delays and RF filters [85, 121]. Systems such as beamforming devices [112] can also be improved in quality factor and angular resolution. Other approaches to filtering include RF bandwidth scaling [125] that yields a particular bandwidth for each wavelength channel, with the total bandwidth (maximum RF signal bandwidth) will depend on the number of wavelengths which is dramatically increased with micro-combs.

Recently [121], we reviewed transversal filtering and bandwidth scaling methods based on Kerr micro-combs, as applied to RF and microwave spectral filters followed by a review of temporal based signal processing [129]. In this paper, we review recent progress in microwave and RF photonic based channelizers based on Kerr micro-combs. We cover devices achieved with both widely spaced micro-combs with an FSR of 200GHz [113] as well as results obtained with record low FSR micro-combs with a spacing of $49 \mathrm{GHz}$, operating via soliton crystals [122]. We highlight their potential and future possibilities, contrasting the different methods and use of the differently spaced micro-combs. While 200GHz Kerr microcombs have been successful for RF channelizers, achieving high levels of performance, high versatility and dynamic reconfigurability, their large comb spacing limited the number of channels as well as the RF bandwidth that can be achieved without the use of thermal tuning. This is an important consideration because RF channelizers need many components such as optical amplifiers and spectral shapers that are only available at telecom wavelengths (1530-1620nm). The limitation in the number of channels has restricted the overall RF bandwidth, frequency resolution, and dynamic reconfigurability of micro-comb based RF channelizers. To overcome this, we focused on RF channelizers based on record low FSR combs, with a spacing of $49 \mathrm{GHz}$, in order to achieve over 90 channels in the C-band [122]. This represents the highest for any micro-comb based RF channelizer. Our results confirm the feasibility of achieving high performance 
reconfigurable transversal RF filters for signal processing with reduced footprint, complexity, and cost. We first review the microcombs used to realize the RF channelizers, followed by the results for $200 \mathrm{GHz}$ microcomb based channelizers and finally by the $49 \mathrm{GHz}$ soliton crystal based systems.

\section{KERR MICRO-COMBS}

The formation of micro-combs is a complex process that arises from a combination of a high nonlinear parameter together with a low nonlinear loss as well as a low linear loss, and finally with careful engineering of the dispersion. Microcombs have been realized in a variety of material platforms [47] including magnesium fluoride, silicon nitride, silica, and doped silica glass [47, 70, 85]. In 2008, [39] efficient FWM operating at low (milliwatt) CW power levels was reported in a MRR with a $575 \mathrm{GHz}$ FSR spacing, in a ring resonator with a low Q-factor of around 60,000. This was the first report of any form of nonlinear optics at milliwatt CW power levels in a silica glass-based platform. In 2010 this was followed by the first report of an integrated Kerr micro-comb [37, 38]. Another key breakthrough came in 2017 [116, 117] with reports of integrated Kerr micro-combs with record small FSR's under 50GHz. This significantly increased the available number of channels, or wavelengths, to more than 80 in the telecommunications wavelength $C$ band from $1530-1565 \mathrm{~nm}$. In addition to their low FSR spacings, these Kerr micro-combs operated in a different mode to DKS states [66-73], in a process that has been called soliton crystals [130, 131]. Many innovative states have been reported in micro-combs, including microcombs with low threshold powers [132], dark solitons [133], laser-cavity solitons [134] and others [135-140].

The devices that were used in the work reviewed here were fabricated in Hydex glass [37, 38], a CMOS compatible platform categorized as high index doped silica. Micro-ring resonators with $\mathrm{Q}$ factors ranging from 60,000 to more than 1.5 million have been achieved, with radii ranging from $592 \mu \mathrm{m}$ down to $48 \mu \mathrm{m}$, corresponding to FSRs from 49 - 575 GHz. The RF signal processors used as the basis for the work reviewed here were fabricated in MRRs with FSR spacings of $200 \mathrm{GHz}$ and $49 \mathrm{GHz}$. Films of Hydex glass $(n=\sim 1.7$ at $1550 \mathrm{~nm}$ ) were deposited via PECVD and photolithographically patterned with deep ultraviolet stepper mask aligners. Waveguides with very low surface roughness were created by reactive ion etching, after which an upper cladding of silica glass $(n=\sim 1.44$ at $1550 \mathrm{~nm})$ was grown. We use both lateral and vertical bus-ring coupling schemes, with a gap typically of about $200 \mathrm{~nm}$. Vertical coupling can be controlled by film growth more accurately than by lithography. Hydex glass has many advantages including a very low linear loss $(\sim 0.06$ $\left.\mathrm{dB} \cdot \mathrm{cm}^{-1}\right)$, a reasonably large nonlinear parameter $\left(0.233 \mathrm{~W}^{-1} \cdot \mathrm{m}^{-1}\right)$, and probably most importantly, negligible TPA even up to many gigawatts $\mathrm{cm}^{-2}$ of power. We achieved high $\mathrm{Q}$ factor resonators of $\sim 1.5$ million for both $49 \mathrm{GHz}$ and $200 \mathrm{GHz}$ spaced MRRs. After packaging with fiber pigtails, the coupling loss can be as low as $0.5 \mathrm{~dB}$ per facet with the aid of onchip mode converters.

For the $200 \mathrm{GHz}$ devices, combs were generated by amplifying the $\mathrm{CW}$ pump power to more than $1 \mathrm{~W}(>+30 \mathrm{dBm})$. Next the wavelength was tuned from blue to red relative to the resonance wavelength, targeting a TE resonance near $\sim 1550$ $\mathrm{nm}$. As the offset between the cold resonance and pump wavelength became sufficiently small the intracavity pump power reached a threshold when modulation instability gain yielded oscillation [47], first generating primary combs with its spacing determined by the MI gain peak, which is a function of dispersion as well as the intra-cavity pump power. As the detuning changed further, microcombs with a spacing equal to the FSR appeared. While these were not soliton states, nonetheless we found that they were more than adequate for our applications to microwave signal processing. We found that it was not necessary to strictly operate in soliton states such as the dissipative Kerr solitons (DKS) [66]. This is an important issue since, while much progress has now been made around DKS solitons [140], they nonetheless intrinsically require quite complicated pump tuning dynamics, generally employing both simultaneous amplitude (power "kicking") and wavelength sweeping, including in reverse directions, to be able to "kickstart" the solitons from out of the chaos. Our early work on micro-combs was based on these $200 \mathrm{GHz}$ FSR combs that operated in this partially coherent state. As mentioned, while not rigorous soliton states, they were still low noise enough and managed to avoid the chaotic regime [47] - we found them to be more than adequate for our RF work. They were used to successfully demonstrated many different RF signal processing functions [85, 107, 109 -111].

More recently, we have used soliton crystal microcombs that featured not only this new mode of operation, but with a record low FSR spacing of $49 \mathrm{GHz}[130,131]$. Soliton crystals arise from mode crossings and are easier and more reliable to generate than other solitons, including DKSs, and even easier than the partially coherent states of the $200 \mathrm{GHz}$ resonators. They can be generated even with simple manual control of the pump wavelength and power, without any complex pump dynamics. The underlying physics for this is based on the fact that the internal optical energy in the cavity of the soliton crystals is very close to that of the chaotic state. Hence, when soliton crystals are generated from chaotic states, there is 
only a small net change in the intracavity optical energy. This means that there is virtually no induced shift in the resonant wavelength - either thermally or via the Kerr nonlinear effect. It is this shift in resonant wavelength and internal energy that makes DKS states so difficult to generate since the resonance "avoids' the pump wavelength via the self-induced shifting. This same effect is also responsible for greatly increasing the efficiency of the soliton crystals, so that the energy in the comb lines are much higher relative to the pump power than the DKS states, particularly for single soliton DKS states. There is one drawback of soliton crystal states, however, and that is that their spectra are not flat - they have characteristic nonuniform "curtain" patterns. While this can sometimes mean that spectral flattening is required, this is not always the case. Indeed, this issue has not prevented soliton crystals from achieving many breakthroughs in high performance photonic RF microwave functions. Even though the resonator mode-crossing needs to be engineered, as well as anomalous dispersion, these issues have not posed a significant barrier and high fabrication yields have been achieved [64]. The specific micro-comb states reached was not important for RF applications, only that low RF noise and high coherence be achieved, and we found this to be easy to generate with simple pump wavelength tuning. Soliton crystals have yielded the lowest noise levels of any of the micro-comb states that we have worked with and so we have focused on them for our RF work, featuring a low phase-noise microwave oscillator [120].

\section{PHOTONIC RF AND MiCROWAVE CHANNELIZERS}

The ability to detect and analyze RF and microwave signals with very large bandwidths is critical for radar systems, electronic warfare, satellite communications and more [141-150]. RF and microwave channelizers, which effectively slice the RF spectrum into many frequency bands that are compatible with digital processing [151], are attractive for RF signal detection and analysis. Conventional RF channelizers usually use an array of RF filters and so are susceptible to the electronic bandwidth limitation. Photonic methods are promising for RF channelizers since they can achieve high bandwidths and also have very strong electromagnetic interference immunity.

Early reports of RF photonic channelizers used methods where RF signals were transmitted over single optical wavelengths and were then physically split to achieve spectral channelizing, usually with diffraction gratings [12], acoustooptic crystals [153], fiber Bragg gratings [154], or integrated photonic chips [155]. However, all of these methods require many narrow linewidth and spectrally dense, precisely centered, filters. Hence these systems tend to be limited in spectral resolution as well as in the number of RF channels. They also tend to have a large footprint.

Recent work on RF spectral slicing using multicasting of RF signals onto many optical wavelengths simultaneously has adopted a number of techniques including stimulated Brillouin scattering [156-158], parametric nonlinear optical processes in fiber [159, 160], spectrally incoherent sliced sources [161], discrete laser arrays [162], or electro-optic modulator generated frequency combs $[151,163,164]$. Other approaches that use wavelength scanning devices [165] or dispersive Fourier transformations [166] have also been reported, but these generally face limitations of one sort or another, including limited channel numbers and limited RF spectral resolution. They also tend to be fairly complex and expensive because of the requirement for many components such as external RF sources and mode-locked lasers etc.. Integrated Kerr microcombs [36-38, 47, 167], particularly those fabricated in CMOS compatible platforms [37- 47, 49 - 61, 168, 169], have many advantages for broadband RF channelizers compared with discrete multi-wavelength sources, such as being able to provide a much higher number of channels or wavelengths [107-129, 170] with a greatly reduced footprint and lower complexity.

Here, we review recent work on broadband photonic RF channelizers realised through the use of integrated optical frequency Kerr microcombs, in combination with passive ring resonator filters. By using an on-chip Kerr comb source consisting of an active nonlinear micro-ring resonator (MRR) with an FSR spacing of either $200 \mathrm{GHz}$ or $49 \mathrm{GHz}$, in combination with a passive on-chip MRR based filter with a free spectral range (FSR) of $49 \mathrm{GHz}$ and Q factor of $1.55 \times 10^{6}$, we achieved RF channelizers with high performance. The use of a $49 \mathrm{GHz}$ comb resulted in a larger number of channels since the FSR of the comb source was nearly matched to the $49 \mathrm{GHz}$ passive MRR. For the $200 \mathrm{GHz}$ spaced comb, only every $4^{\text {th }}$ resonance of the $49 \mathrm{GHz}$ passive MRR could be used. We verified the RF performance experimentally at frequencies up to $26 \mathrm{GHz}$, achieving a high spectral resolution of better than $1.04 \mathrm{GHz}$ for both systems. In addition to high RF performance, this approach offers a reduced footprint, lower complexity, and potentially lower cost. 

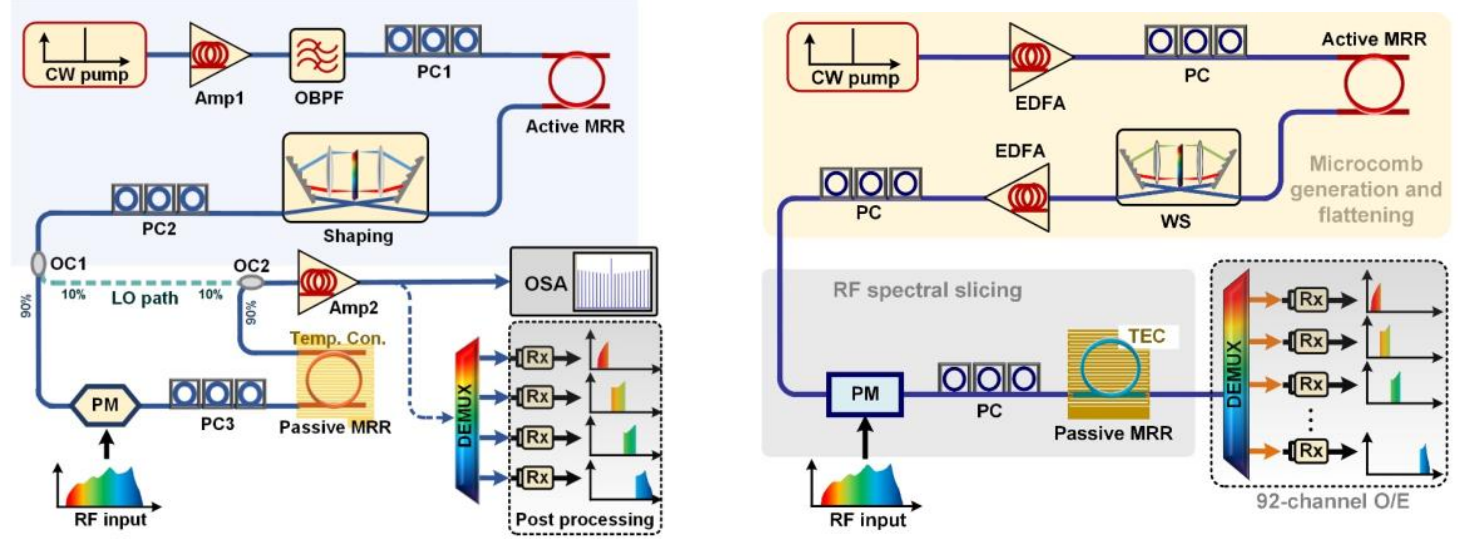

Fig. 1. Schematic diagram of the broadband RF channelizer based on an integrated optical comb source. Amp: erbium-doped fibre amplifier. OBPF: optical bandpass filter. PC: polarization controller. MRR: micro-ring resonator. OC: optical coupler. PM: phase modulator. Temp. Con.: temperature controller. DEMUX: de-multiplexer. Rx: Receiver. OSA: Optical spectrum analyzer. Right: Schematic diagram of the broadband $\mathrm{RF}$ channelizer based on a soliton crystal microcomb. EDFA: erbium-doped fibre amplifier. PC: polarization controller. MRR: micro-ring resonator. WS: Waveshaper. PM: phase modulator. TEC: temperature controller. DEMUX: de-multiplexer. Rx: Receiver.

\section{PRINCIPLE OF OPERATION}

Figure 1 shows a schematic of the RF channelizer operating by combining an active microcomb as a source (in our case combs with FSRs of either $200 \mathrm{GHz}$ or $49 \mathrm{GHz}$ ) with a $49 \mathrm{GHz}$ passive MRR that acts as a narrow optical filter. We first discuss the system based on the 200GHz FSR microcombs, where Kerr combs were generated in the MRR by pumping by a tunable CW laser, amplified by an erbium-doped fibre amplifier. A tunable optical bandpass filter suppressed the amplified spontaneous emission noise from the amplifier, and a fibre polarization controller was used to adjust the polarization to optimize the coupled power into the waveguide. When the pump wavelength was tuned near a resonance of the active MRR, then if the power was sufficiently high, parametric gain would eventually result in optical parametric oscillation. Lastly, Kerr combs with a spacing equal to the MRR FSR were generated $\delta_{\mathrm{OFC}}(\sim 200 \mathrm{GHz})$. In the channelizer wavelength range, the frequency of the $k_{t h}(k=2,3,4, \ldots)$ comb line is

$$
f_{\mathrm{OFC}}(k)=f_{\mathrm{OFC}}(1)+(k-1) \delta_{\text {OFC }}
$$

where $f_{\mathrm{OFC}}(1)$ is the first comb line frequency on the red side. The combs were then flattened by a Waveshaper and passed through a phase modulator (PM) in order to multicast the broadband RF signal onto all wavelengths. Finally, all of the 200 $\mathrm{GHz}$ comb lines, each imprinted with the RF signal spectrum, were spectrally sampled, or sliced, by the second passive high-Q MRR that had an FSR of $49 \mathrm{GHz}$. Hence, in the first series of experiments we had to use every $4_{\text {th }}$ resonance of the passive MRR to achieve the spectral slicing, which resulted in an effective FSR with a spacing $\delta_{\text {MRR }}$ of $\sim 196 \mathrm{GHz}$. Therefore, the RF spectrum on each of the $200 \mathrm{GHz}$ microcomb lines was progressively sampled with a sequential step between resonances of about $4 \mathrm{GHz}$. The output channelized $\mathrm{RF}$ frequencies are:

$$
\begin{aligned}
& f_{\mathrm{RF}}(k)=f_{\mathrm{MRR}}(k)-f_{\mathrm{OFC}}(k) \\
& =\left[f_{\mathrm{MRR}}(1)-f_{\mathrm{OFC}}(1)\right]+(k-1)\left(\delta_{\mathrm{MRR}}-\delta_{\mathrm{OFC}}\right)
\end{aligned}
$$

where $f_{\mathrm{RF}}(\mathrm{k})$ is the $k_{t h}$ channelized RF frequency, $f_{\mathrm{MRR}}(\mathrm{k})$ is the $k_{t h}$ centre frequency of the filtering MRR. Here, [ $f_{\mathrm{MRR}}(1)-$ $\left.f_{\mathrm{OFC}}(1)\right]$ is the relative spacing between the first comb line and adjacent filtering resonance, corresponding to the channelized RF frequency offset, and ( $\left.\delta_{\mathrm{OFC}}-\delta_{\mathrm{MRR}}\right)$ corresponds to the channelized RF frequency step between adjacent wavelengths. While the RF channels could in principle be sampled with an optical demultiplexer such as an arrayed waveguide grating, as long as it had a channel spacing that matched the FSR (or a multiple of) of the $49 \mathrm{GHz} \mathrm{MRR}$, in practice AWG multiplexers and similar devices generally do not have nearly as fine a resolution as the MRRs used here they tend to have channel widths matched to the ITU grid of $50 \mathrm{GHz}$ or $100 \mathrm{GHz}$, versus the MRRs used here that had a $3 \mathrm{~dB}$ linewidth of about $1 \mathrm{GHz}$. The RF signals were then detected via homodyne detection, where the flattened comb lines were first separated out before phase modulation in order to serve as local oscillators (LO). They were then coupled together with the channelized RF optical sidebands and coherently detected for post processing. In the initial work based on the $200 \mathrm{GHz}$ combs [113], however, we only measured the broadband optical spectrum and not the actual RF waveform, 
a

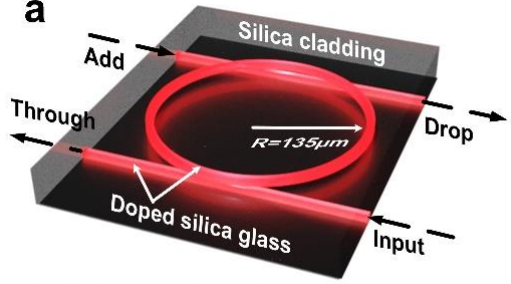

Active MRR with $135 \mu \mathrm{m}$ radius b

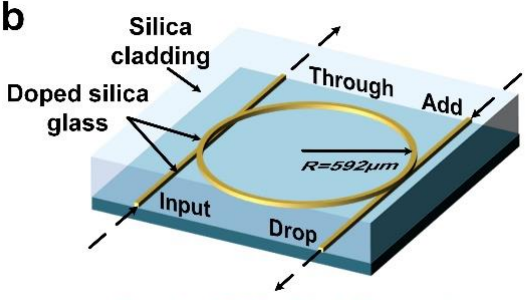

Passive MRR with $592 \mu \mathrm{m}$ radius

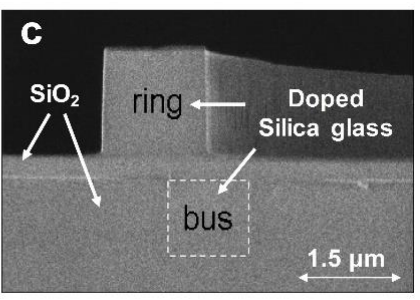

$1.5 \mu \mathrm{m}$

Fig. 2. Schematic illustration of the (a) $200 \mathrm{GHz}-\mathrm{FSR}$ MRR and (b) $49 \mathrm{GHz}-\mathrm{FSR}$ MRR. (c) SEM image of the cross-section of the $200 \mathrm{GHz}$ MRR before depositing the silica upper cladding.

to verify the feasibility of our approach, deferring channel demultiplexing and RF detection to later work. Regardless, however, even in that initial work the large number of wavelengths generated by the micro-comb resulted in a large number of channels, with broadband RF channelizing with digital-compatible channel bandwidths.

That first work was followed by an RF channelizer based on a $49 \mathrm{GHz}$ microcomb combined with the $49 \mathrm{GHz}$ passive MRR filter [122] that resulted in a photonic RF channelizer with significantly improved performance. This was a result of the fact that the two MRRs had quasi-matched FSRs, both near 49GHz. The first MRR generated a soliton crystal microcomb while the second MRR acted as a passive filter. Using the finer spaced (than the original $200 \mathrm{GHz}$ ) microcomb had two significant benefits. First, the much smaller FSR of the comb source generated as many as 92 wavelength channels across the $\mathrm{C}$ band (versus 20 in the initial work). This resulted in a much larger instantaneous operational bandwidth of $8.08 \mathrm{GHz}$ without any thermal tuning - more than 22 times larger than the 200GHz comb device [113]. Secondly, by using an integrated high-Q MRR with a (roughly) matching FSR to slice the RF spectrum via the Vernier effect, an RF channelization step of only $87.5 \mathrm{MHz}$ was achieved. This effectively resulted in continuous RF spectrum channelization since the channel step was comparable to (slightly smaller than) the passive MRR spectral resolution of $121.4 \mathrm{MHz}$. Finally, another difference with the second device over the first one is that in this device we used parallel phase-modulation to intensity-modulation (PM-IM) conversion across all of the wavelength channels. This directly yielded an RF output in a compact and stable scheme without any need for separate local oscillator paths. Finally, the channelizer's operation frequency range could be dynamically tuned by adjusting the offset frequency between the microcomb and passive MRR. By thermally tuning the passive MRR we succeeded in achieving RF channelization over a very large RF range of 17.55 GHz. In addition to high RF performance, this approach offers a lower complexity, reduced footprint, and even lower cost.

The broadband RF channelizer (Fig. 2 (b)) is very similar to the previous device and also consists of three modules. The first is the microcomb generation and flattening, where an active MRR was pumped by a CW laser to initiate parametric oscillation. With the MRR's high Q factor of $>10^{6}$ million, together with the high nonlinear FOM and engineered anomalous dispersion, enough parametric gain was generated to produce Kerr frequency combs. The nature of the oscillation state of the microcomb was determined primarily by the pump to resonance detuning together with the pump power. By sweeping the pump wavelength from blue to red, a range of dynamic nonlinear states could be achieved, including coherent soliton states. The comb lines can also be labelled by Eqs $(1,2)$ except that now the number of lines is much larger. $N$ microcomb lines are generated with a spacing of $\delta_{\mathrm{OFC}}$, the optical frequency of the $k_{t h}(k=1,2,3, \ldots, 92)$ comb line is given by $\mathrm{Eq}(1)$. An optical Waveshaper was again used to flatten the comb line powers.

In the second module, the flattened comb lines were passed through an electrooptical phase modulator, where the input broadband RF signal was multicast onto all wavelengths. Next, as before the replicated RF spectra were sliced by the passive MRR with an FSR of $\delta_{\mathrm{MRR}}$, where the slicing resolution is given by the $3 \mathrm{~dB}$ bandwidth of the passive resonator, about $1 \mathrm{GHz}$ in our case. As a result, the RF spectral segments on all wavelength channels were effectively channelized with a progressive $\mathrm{RF}$ centre frequency given by $\mathrm{Eq}(2)$.

For the $49 \mathrm{GHz}$ comb based device, we employed phase modulation and notch filtering (i.e., using the transmission through port of the passive MRR) to convert from phase to intensity modulation. Phase modulation first produced both lower and upper sidebands that had opposite phases. One of the sidebands was then rejected by the notch resonance, leaving the optical carrier to beat with the other unsuppressed sideband on photodetection. Thus, this process resulted in converting the modulation from phase to single-sideband intensity modulation without a local oscillator. Hence, $N$ bandpass filters were achieved, each with a spectral bandwidth resolution $\Delta f$, determined by the passive MRR's resonant linewidth. The center $\mathrm{RF}$ frequencies $f_{\mathrm{RF}}(\mathrm{k})$ were determined by the spacing between the optical carriers and the passive resonances $\left[f_{\mathrm{MRR}}(\mathrm{k})-f_{\mathrm{OFC}}(\mathrm{k})\right]$, as described in Eq. (2). Therefore, the input RF spectrum was demultiplexed, or channelized, into $N$ segments, each at $f_{\mathrm{RF}}(\mathrm{k})$ and each having a bandwidth of $\Delta f$. 

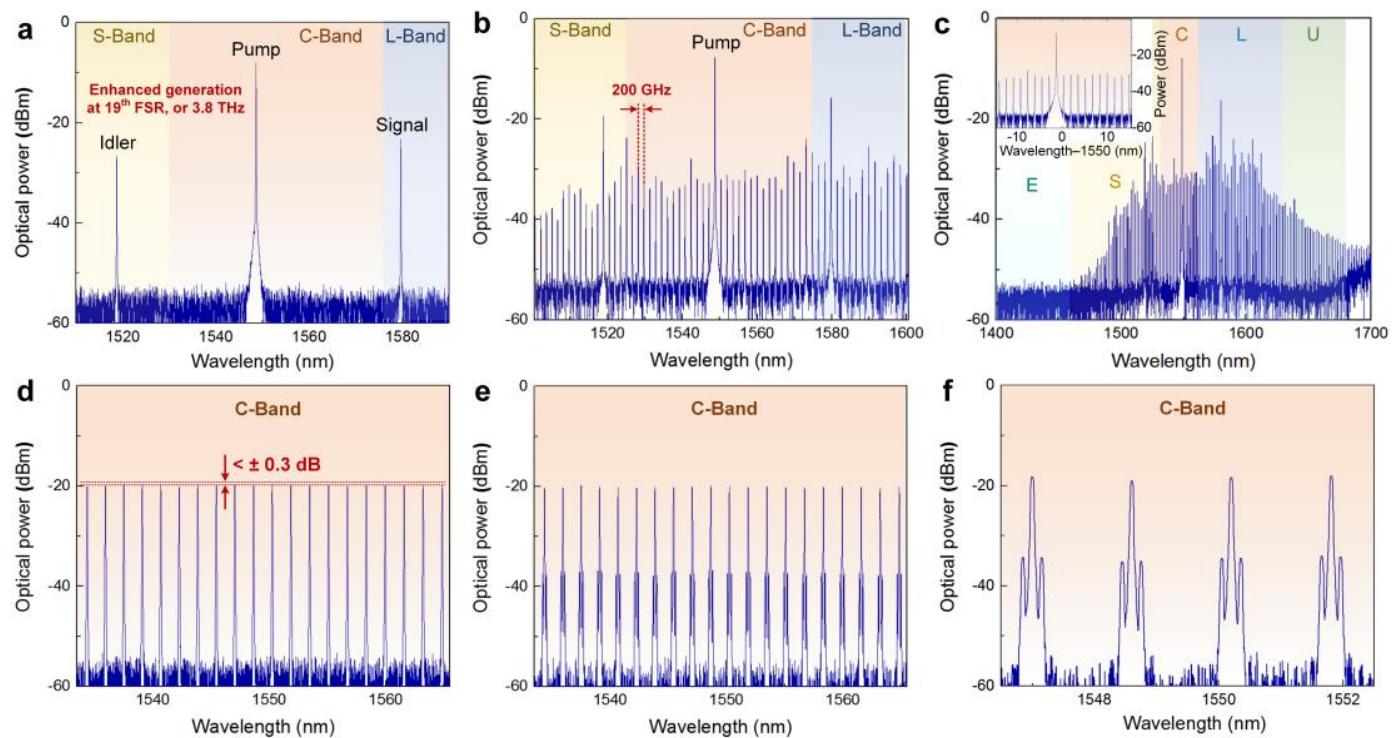

Fig. 3. Optical spectrum for the $200 \mathrm{GHz}$ FSR micro-comb device. (a) the primary comb, (b) the secondary comb, (c) the Kerr comb with $300 \mathrm{~nm}$ span, (d) the shaped optical comb for the channelizer with less than $0.5 \mathrm{~dB}$ unflatness, (e) 20 and (f) selected $4 \mathrm{comb}$ lines modulated by RF signals.

As mentioned, this approach did not require any other physical local oscillator paths to achieve coherent homodyne detection, and so is much more compact and stable than those involving interfering paths [113]. Finally, the wavelength channels were de-multiplexed and converted back into the electrical domain separately via an array of photodetectors, simultaneously yielding $N$ channelized RF signals, each with a spectral width of $\Delta f$, typically within the operation bandwidth of ADCs. After that, the channelized RF signals were converted into digital signals via an ADC array and processed with digital domain tools.

\section{200GHz Microcomb System: ResUlts}

Both MRRs (Fig. 2) were fabricated in a high-index doped silica glass platform using CMOS compatible fabrication processes. First, high-index $(\mathrm{n}=\sim 1.70$ at $1550 \mathrm{~nm}$ ) Hydex glass films were deposited using standard plasma enhanced chemical vapour deposition (PECVD), then patterned photo-lithographically and etched via reactive ion etching to form waveguides with exceptionally low surface roughness. Finally, silica glass $(\mathrm{n}=\sim 1.44$ at $1550 \mathrm{~nm})$ or Hydex with the equivalent refractive index, was deposited via PECVD as an upper cladding. The advantages of our platform for nonlinear OPOs include ultra-low linear loss $\left(\sim 0.06 \mathrm{~dB} \cdot \mathrm{cm}^{-1}\right)$, a moderate nonlinearity parameter $\left(\sim 233 \mathrm{~W}^{-1} \cdot \mathrm{km}^{-1}\right)$, and, in particular, a negligible nonlinear loss up to extremely high intensities $\left(\sim 25 \mathrm{GW} \cdot \mathrm{cm}^{-2}\right)$ [38]. The low linear loss resulted in ring resonator $Q$ factors of $>1 \times 10^{6}$. A scanning electron microscope (SEM) image of the cross-section of the $200 \mathrm{GHz}$ MRR before depositing the $\mathrm{SiO}_{2}$ upper cladding is shown in Fig. 2(c). The radii of the active $200 \mathrm{GHz}$ MRR (for comb generation) and the passive MRR (for spectral slicing) were $\sim 135 \mu \mathrm{m}$ and $\sim 592 \mu \mathrm{m}$, corresponding to FSRs of $\sim 1.6 \mathrm{~nm}(\sim 200 \mathrm{GHz}$ ) and $\sim 0.4 \mathrm{~nm}(\sim 49 \mathrm{GHz})$, respectively. After packaging the device with fibre pigtails, the total fibre-fiber through-port insertion loss was $\sim 3.5 \mathrm{~dB}$ for the $200 \mathrm{GHz}$ MRR and $\sim 1.5 \mathrm{~dB}$ for the $49 \mathrm{GHz}$ MRR.

To generate Kerr combs, the pump power was amplified to $\sim 500 \mathrm{~mW}$ and the wavelength swept from blue to red. When the pump wavelength was tuned close to the resonance of the active MRR, primary combs were generated (Fig. 3(a)). Signal and idler lines were generated in the S-band and L-bands respectively, and the spacing between the signal and pump was $19 \mathrm{FSR}$, or $3.8 \mathrm{THz}$, determined by the parametric gain curve. When the detuning between the pump wavelength and the adjacent resonance was further changed, the parametric gain lobes broadened and secondary comb lines with a spacing equal to the FSR appeared via cascaded four wave mixing. Finally, flat Kerr combs were generated with the pump wavelength set to $1548.58 \mathrm{~nm}$. As shown in Fig. 3(b-c), the resulting Kerr comb was over 200-nm wide, covering four bands (S, C, L, U) and was relatively flat over the full $\mathrm{C}$ and $\mathrm{L}$ bands, thus enabling a record (at the time) large number of wavelengths ( 20 over the C-band and > 60 over the C/L-bands). The large Kerr comb spacing also yielded an increased Nyquist zone, corresponding to an RF bandwidth of $>100 \mathrm{GHz}$. This bandwidth is extremely challenging for mode-locked lasers and externally-modulated comb sources to achieve. 

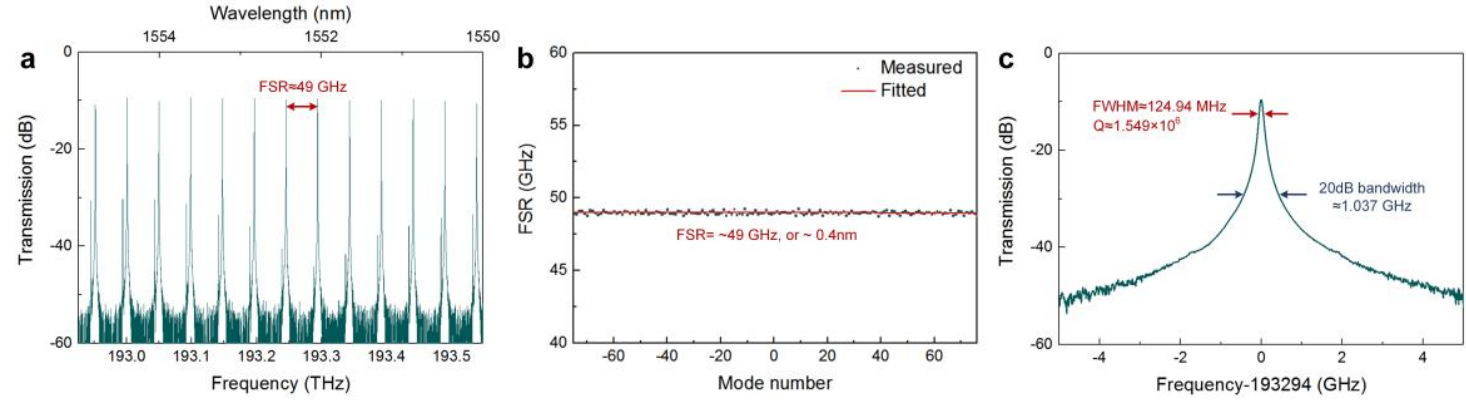

Fig. 4. Drop-port transmission spectrum of the passive on-chip $49 \mathrm{GHz}$ MRR (a) with a span of $5 \mathrm{~nm}$, (b) showing an FSR of $49 \mathrm{GHz}$, and (c) a resonance at $193.294 \mathrm{THz}$ with full width at half maximum (FWHM) of $124.94 \mathrm{MHz}$, corresponding to a $\mathrm{Q}$ factor of $1.549 \times 10^{6}$.

While the spectral profile of the $200 \mathrm{GHz}$ comb was not indicative of operation in the single cavity soliton regime, we did not experience any significant limitations as a result. Theoretical analysis of our observations revealed that the Kerr comb was working under "partially coherent" conditions that featured stable phase and amplitude. In fact, the spatial patterns of what we observed appear to be similar to the so called "soliton molecules" [130,131,171-173], which feature reasonably low intensity noise comparable with that of cavity solitons.

We note that since the comb source could serve as both the optical carrier for RF sideband generation as well as the LO for $\mathrm{RF}$ receiving (in the case of the $200 \mathrm{GHz}$ comb device), the channelized RF optical sidebands and LOs were inherently coherent, and thus strictly phase-locked comb lines are not necessary - which, in fact, is an advantage when comparing with RF coherent receivers [174]. Similarly, the coherence between adjacent WDM channels is also not required since the different lines do not beat against one another. The 20 channels over the C-band of the $200 \mathrm{GHz}$ Kerr comb were then shaped by a Waveshaper (Finisar 4000S) to get uniform channel weights. A feedback control path was used to accurately read and shape the comb lines' power, first detected by an optical spectrum analyser and then compared with the ideal weights (uniform in our case). This allowed us to generate a feedback error signal to the waveshaper to calibrate the system and achieve accurate comb shaping and flatness of the comb envelope to within $\pm 0.3 \mathrm{~dB}$ (Fig. 3(d)). Note that in practice passive gain flattening filters can be used in place of the Waveshaper to flatten the spectrum. The phase modulator was then used to multicast the RF signal onto each comb line. Figures 3(e-f) show the optical spectra of the flat comb lines modulated by the RF signal. The 20 equalized channels were limited to the $\mathrm{C}$-band purely by the operational range of the waveshaper. In principle the number of wavelengths could easily be increased up to 60 by using a $C+L$ band waveshaper. Next, the multicast RF signal on each wavelength channel was spectrally sliced by the passive $49 \mathrm{GHz}$ MRR. Figures. $4(\mathrm{a}-\mathrm{b})$ show the drop-port transmission of the $49 \mathrm{GHz}$ MRR over a $5 \mathrm{~nm}$ range, with an FSR of $\sim 49 \mathrm{GHz}(\sim 0.4 \mathrm{~nm})$ over 152 modes (denoting the mode at $193.294 \mathrm{THz}$ as 0). The transmission of a single resonance (Fig. 4(c)) shows a full width

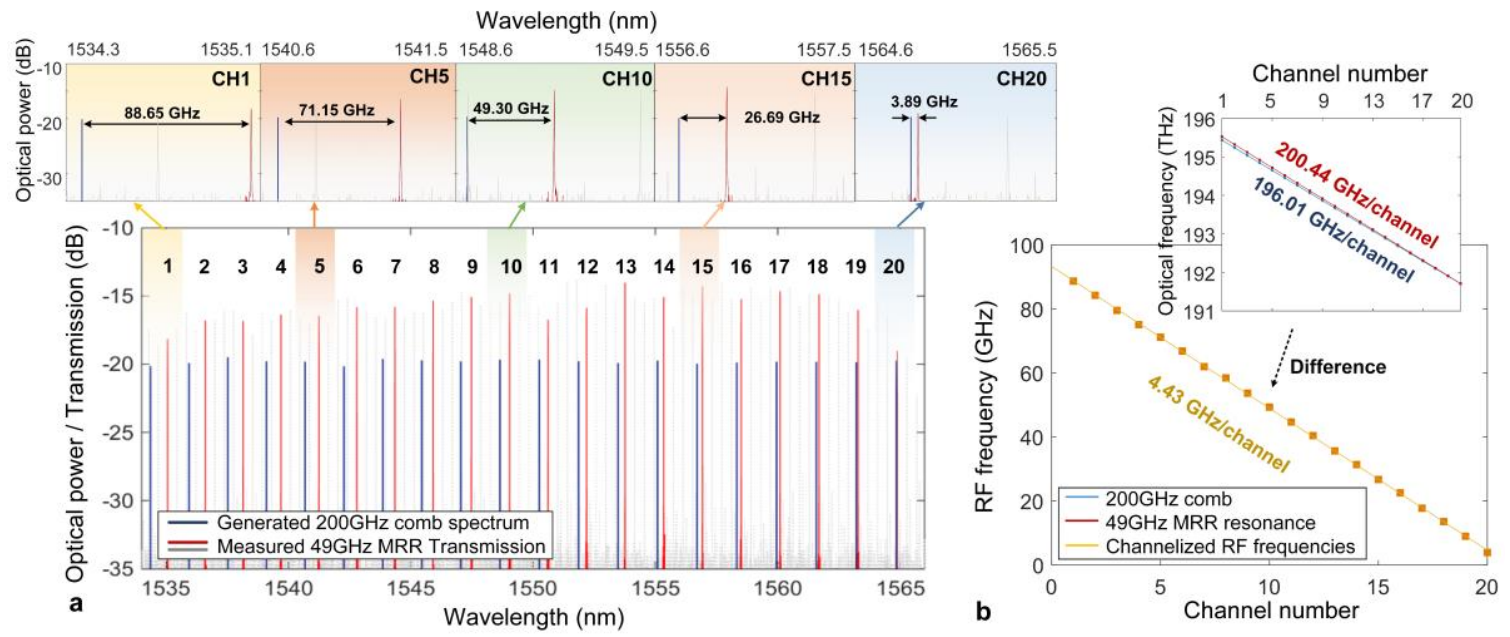

Fig. 5. (a) The measured optical spectrum of the $200 \mathrm{GHz}$ micro-comb and transmission of $49 \mathrm{GHz}$ MRR. Zoom-in views of the channels with different channelized RF frequencies. (b) Extracted channelized RF frequencies, inset shows corresponding optical frequencies of the comb lines and spectral slicing resonances. 

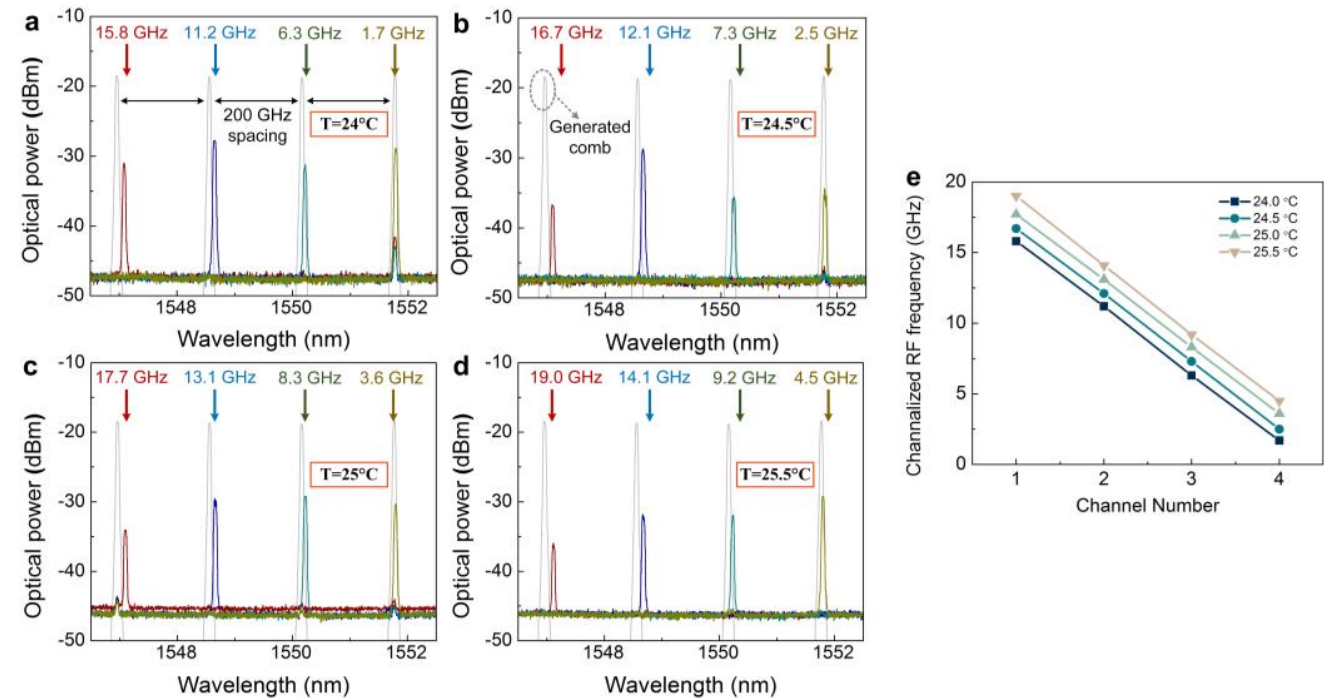

Fig. 6. RF response of $200 \mathrm{GHz}$ Channelizer. Measured optical spectrum of $49 \mathrm{GHz} \mathrm{MRR}$ 's output with different input RF frequencies, with the temperature of the $49 \mathrm{GHz}$ MRR set to (a) $24.0^{\circ} \mathrm{C}$, (b) $24.5^{\circ} \mathrm{C}$, (c) $25.0^{\circ} \mathrm{C}$, and (d) $25.5^{\circ} \mathrm{C}$. (e) Channelized RF frequencies at different wavelength channels with different temperatures.

maximum (FWHM) of $124.94 \mathrm{MHz}$, corresponding to a $\mathrm{Q}$ factor of $1.549 \times 10^{6}$, and a $20 \mathrm{~dB}$ bandwidth of $1.04 \mathrm{GHz}$, which determines the RF resolution. This RF resolution is compatible with state-of-the-art analog-to-digital converters [143], thus leading to digital-compatible broadband RF channelization.

To illustrate the operation principle of the RF channelizer, we show the $200 \mathrm{GHz}$ comb spectrum and transmission of the $49 \mathrm{GHz}$ passive MRR (highlighting every fourth resonance that aligns with the $200 \mathrm{GHz}$ comb) in Fig. 5(a). Fig. 5(a) shows that for the $20 \mathrm{comb}$ channels over the C-band, the relative offset between the $200 \mathrm{GHz}$ comb lines and every fourth $49 \mathrm{GHz}$ MRR resonance yields the RF spectral sliced frequency, which varies from 3.89 to $88.65 \mathrm{GHz}$. The relative spacing of the $200 \mathrm{GHz}$ comb and $49 \mathrm{GHz}$ MRR were linearly fit (Fig. 5(b)) obtaining a comb spacing of $\delta_{\mathrm{OFC}}=200.44 \mathrm{GHz}$ and a fit FSR of the spectrally sliced resonances (i. e., $\delta_{\text {MRR }}$ is the frequency spacing between every $4_{\text {th }}$ resonance of the 49 GHz MRR) of $\delta_{\text {MRR }}=196.01 \mathrm{GHz}$, thus resulting in a channelized RF frequency step between successive channels $\left(\delta_{\mathrm{OFC}}\right.$ $\left.-\delta_{\mathrm{MRR}}\right)$ of $4.43 \mathrm{GHz}$.

We measured the RF performance of the channelizer up to $20 \mathrm{GHz}$. This only required 4 of the 20 channels in Figure 5 , corresponding to four RF frequencies from 1.7 to $19.0 \mathrm{GHz}$. We measured the output of the $49 \mathrm{GHz}$ passive MRR with an optical spectrum analyzer (Fig. 6), showing that optical RF sidebands with different RF frequencies were channelized at the different wavelengths. As Fig. 6(a) shows, optical-carrier RF tones at frequencies of $1.7 \mathrm{GHz}, 6.3 \mathrm{GHz}, 11.2 \mathrm{GHz}$, and $15.8 \mathrm{GHz}$ were output on 4 wavelength channels, corresponding to an RF frequency step of $\sim 4.8 \mathrm{GHz}$, in agreement with Fig. 5. The power leakage of channel 4 was mainly due to spurious optical carrier tones, which could be reduced by using an optimized modulation format such as carrier-suppressed single-sideband modulation.

In order to make full use of the RF resolution of our system $(\sim 1 \mathrm{GHz})$ and bridge the gap formed by the $\sim 4.8 \mathrm{GHz} \mathrm{RF}$ step size, we additionally used temperature tuning $[47,174]$ of the passive MRR to continuously control the relative spacing between the source and filtering MRRs' resonances $\left(f_{\mathrm{MRR}}(1)-f_{\mathrm{OFC}}(1)\right)$, with a millisecond thermal response time. Figures $6(\mathrm{a}-\mathrm{d})$ show the output optical spectra at temperatures from $24.0^{\circ} \mathrm{C}$ to $25.5^{\circ} \mathrm{C}$. As reflected in Fig. $6(\mathrm{e})$, the channelized RF frequency offset increased at $\sim 1 \mathrm{GHz} /{ }^{\circ} \mathrm{C}$. By combining the fine resolution temperature tuning with the 4.8 $\mathrm{GHz}$ RF increment across 60 channels over the C/L-bands, this device could achieve a very wide RF bandwidth of greater than $100 \mathrm{GHz}$.

Figure 7 shows the channelized RF frequencies of the different optical channels extracted from Fig. 6, over an RF bandwidth from 1.7 to $19.0 \mathrm{GHz}$, limited by the RF modulator bandwidth. Figure 8 shows the extinction ratio (ER) of the wavelength channels (the optical power ratio of the signal channel to the maximum of other channels) extracted from Figs. $6(\mathrm{a}-\mathrm{d})$, showing up to a $20 \mathrm{~dB}$ ER. The non-uniformity in optical power of the different channels mainly arose from the amplifier spectral gain profile, and this can be greatly reduced with gain flattening filters. The extinction ratio was also limited by excess noise of the second EDFA when adapting the optimized RF modulation format to increase the OSNR. In this case, using lower noise EDFAs would enhance the performance. The thermal controllers of the active and passive 
MRRs had an accuracy of $0.01^{\circ} \mathrm{C}$, contributing to an error of $\sim 50 \mathrm{MHz}$ for the channelized RF frequencies, which could be eliminated by using more precise thermal control or a feedback loop to achieve high performance in

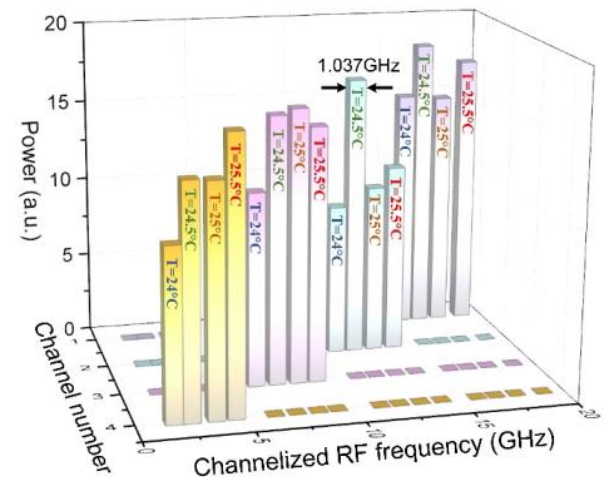

Fig. 7. Channelized RF frequencies at different channels.

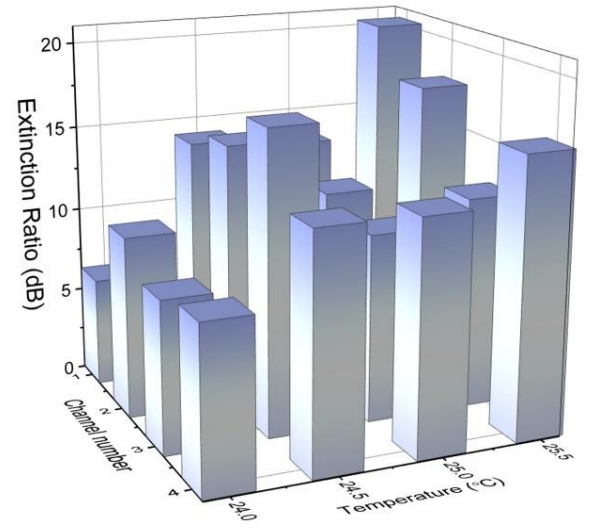

Fig. 8. Extracted extinction ratio of channelized RF signals.
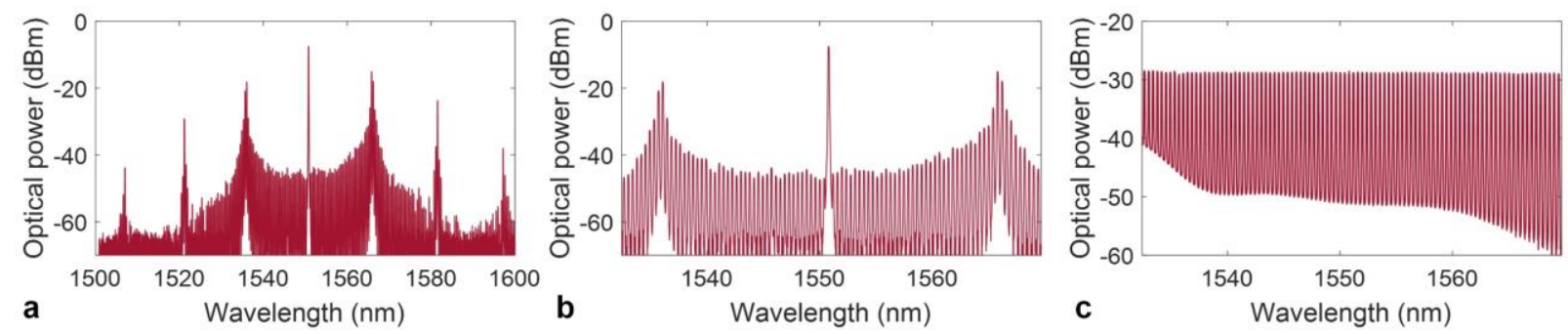

Fig. 9. Optical spectrum of the generated soliton crystal microcomb with (a) $100 \mathrm{~nm}$ and (b) $40 \mathrm{~nm}$ span. (c) Flattened 92 comb lines. 
practical applications. Although employing thermal tuning posed challenges for simultaneous full RF spectrum channelizing, it nonetheless allowed us to demonstrate the capability and the novelty of our method. Thermal tuning is in fact not necessary, provided the RF frequency step between adjacent wavelength channels (i.e., the FSR difference between the active MRR and the passive MRR) is equal to the spectral slicing resolution (i.e, the 20dB bandwidth of the passive MRR), which can be achieved via precise lithographic control of the FSRs of the MRRs. For example, given $\delta_{\mathrm{OFC}}=200$ $\mathrm{GHz}$, then designing the passive MRR such that $\delta_{\mathrm{MRR}}=199 \mathrm{GHz}$, still with a $1 \mathrm{GHz} 20 \mathrm{~dB}$ - bandwidth at the same time. Thus, the full RF spectra could be simultaneously channelized at a resolution of $1 \mathrm{GHz}$ without thermal tuning. While the RF operation bandwidth, given by the product of channel number and resolution, would be slightly less than the device reported here, at $60 \mathrm{GHz}$ for 60 wavelengths (channels) across the $\mathrm{C} / \mathrm{L}$ bands, this could be increased by using a smaller FSR comb (eg., $100 \mathrm{GHz}$ vs. $200 \mathrm{GHz}$ ). Finally, we note that our scheme generates RF output based on homodyne detection, whereas down-conversion of the channelized RF signal would require heterodyne detection where a second optical frequency comb with specially designed FSR and offset is required. Recent advances in dual-comb spectroscopy provide new possibilities for that [163, 175-177]. By employing integrated dual micro-combs with specially designed FSRs and offsets for heterodyne detection [178-180], broadband RF signals can be channelized and down-converted into digital bandwidths for direct analog-to-digital conversion and post processing, thus offering a highly competitive approach for integrated photonic $\mathrm{RF}$ receivers.

\section{49GHZ MiCROCOMB RESULTS}

In this section, we summarize the results for the photonic RF channelizer based on two MRRs with quasi-matched FSRs - both near 49GHz. This approach achieves significantly improved performance over the previous device based on a $200 \mathrm{GHz}$ spaced microcomb. Here, the first MRR generates a soliton crystal microcomb at $49 \mathrm{GHz}$ while the second MRR acts as a passive filter with approximately the same channel spacing. This results in two significant benefits. First, the much smaller overall FSR of the comb source provides up to 92 wavelengths across the $\mathrm{C}$ band. This results in a significantly enhanced instantaneous RF bandwidth of $8.08 \mathrm{GHz}$ - more than $22 \mathrm{x}$ that of the previous device [113]. By using integrated high-Q MRRs with approximately matching spacings to both generate the comb and slice the RF spectrum using a Vernier effect, we achieved a RF channelization step between channels of $87.5 \mathrm{MHz}$. This led to successful continuous channelization of the RF spectrum since the channelization step was smaller than the spectral resolution of 121.4 MHz. We also employed parallel phase-modulation to intensity-modulation (PM-IM) conversion across all wavelength channels - which directly generated an RF output in a stable scheme and compact footprint without needing separate local oscillator paths. Finally, the channelizer's total RF operation frequency range could be dynamically tuned by adjusting the offset frequency between the active microcomb and passive MRR. We thermally tuned the passive MRR to achieve RF channelization over a total bandwidth of $17.55 \mathrm{GHz}$. In addition to achieving high RF performance, our approach offers lower complexity, a reduced footprint, and potentially even lower cost.

Figure $1 b$ shows the setup of the broadband RF channelizer. As before, the microcomb generation was generated by pumping the first MRR with a CW laser to initiate parametric oscillation. The MRR's high > 1 million Q factor together with the high nonlinear figure of merit, and tailored anomalous waveguide dispersion, all contribute to achieving parametric gain to generate the Kerr frequency combs. The state of operation of the frequency comb was determined by the pump to resonance detuning as well as the pump power. By sweeping the pump wavelength from blue to red, diverse nonlinear dynamic states, including the coherent soliton states, could be triggered. As before, for $N$ microcomb lines generated with a spacing of $\delta_{\mathrm{OFC}}$, the optical frequency of the $k_{t h}(k=1,2,3, \ldots, 92)$ comb line is given by Equation (1). Again, an optical spectral shaper (Waveshaper) equalized the channel powers. In the second module, the flattened comb lines were modulated with an electrooptical phase modulator to multicast the RF signal onto all wavelengths. Next, the replicated RF signals imprinted on all comb lines were sampled by the passive MRR with an FSR of $\delta_{\text {MRR, and with a }}$ resolution determined by the $3 \mathrm{~dB}$ bandwidth of the resonator. As a result, the RF spectral segments on all wavelength channels were channelized with a staggered RF frequency, given by Eq. 2.

For this device we used phase modulation and notch filtering (i.e., the transmission of the passive MRR's through port) to achieve phase to intensity-modulation conversion. The phase modulation first yielded upper and lower sidebands with opposite phases, one of which was then suppressed by the notch resonances, leaving the optical carrier and the other unsuppressed sideband to subsequently beat together upon photodetection. Thus, this process effectively converted the modulation format from phase to intensity modulation (single-sideband). As a result, $N$ bandpass filters were realized, each with a spectral resolution $(\Delta f)$ determined by the passive MRR's resonant linewidth. The center RF frequencies $f_{\mathrm{RF}}(\mathrm{k})$ are determined by the spacing between the optical carriers and passive resonances $\left[f_{\mathrm{MRR}}(\mathrm{k})-f_{\mathrm{OFC}}(\mathrm{k})\right]$, as described in Eq. (2). 


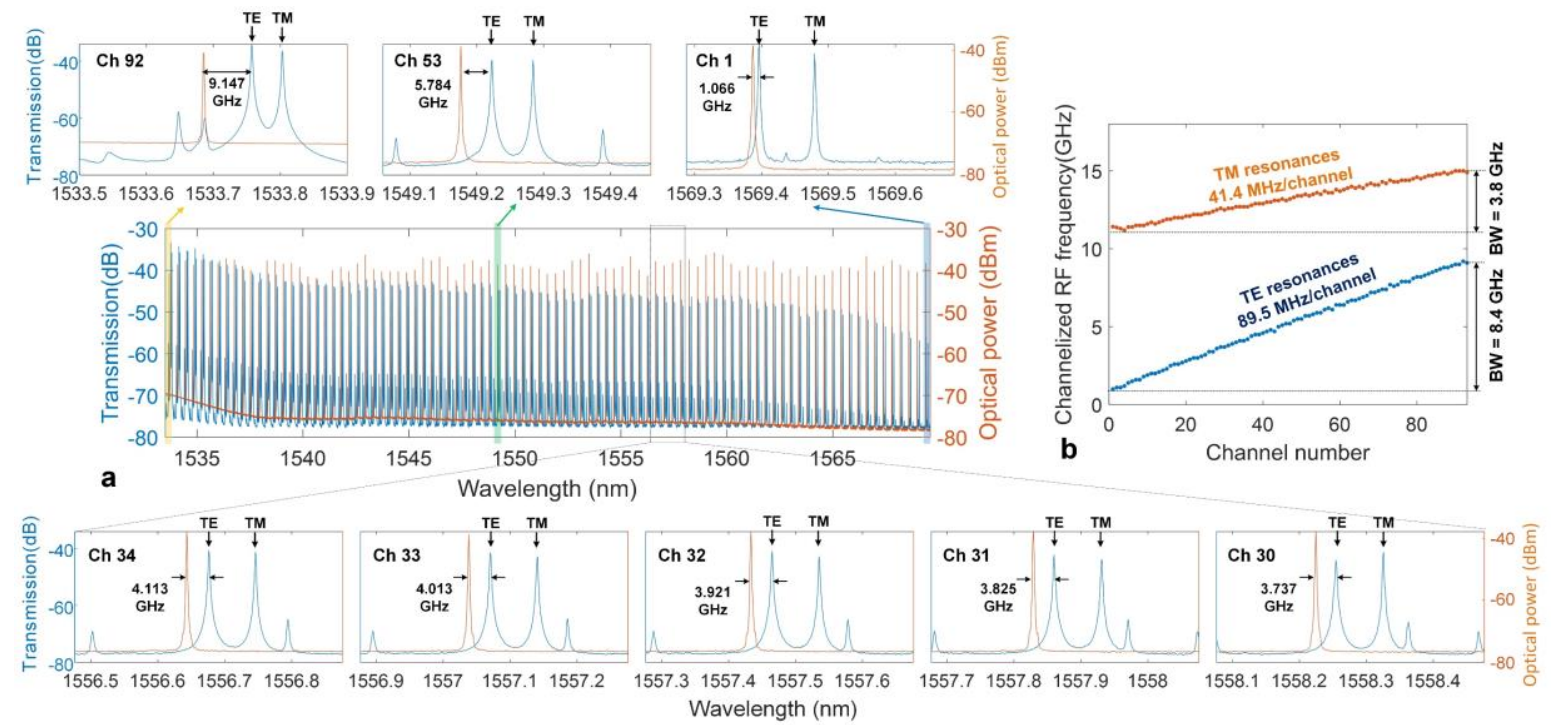

Fig. 10. (a) The measured optical spectrum of the micro-comb and drop-port transmission of passive MRR. (b) Extracted channelized RF frequencies of the 92 channels, calculated from the spacing between the comb lines and the passive resonances. Note that the labelled channelized RF frequencies in (a) is adopted from the accurate RF domain measurement using the Vector Network Analyzer, as shown in the next figure.

As a result, the input RF spectrum is channelized into $N$ segments, each centered at $f_{\mathrm{RF}}(\mathrm{k})$ and with a bandwidth of $\Delta f$. This approach does not require any physical local oscillator paths to achieve coherent homodyne detection, and so is much more compact and stable than the first approach [113]. Finally, the wavelength channels were de-multiplexed and converted back into the electrical domain separately via an array of photodetectors, simultaneously yielding $N$ channelized RF signals, each with a spectral width of $\Delta f$ within the operation bandwidth of ADCs and then converted into digital signals via an array of ADCs and processed with digital domain into digital signals via an array of ADCs and processed with digital domain tools.

The active and passive MRRs were both fabricated in a CMOS-compatible doped silica glass platform, employing fabrication processes as discussed above. The dispersion of the active 49GHz FSR MRR was tailored to be anomalous in the $\mathrm{C}$ band to enable parametric oscillation. Further, a mode-crossing at $\sim 1556 \mathrm{~nm}$ was engineered which could initiate the background wave required for soliton crystal generation. During comb generation, the pump power was boosted to $\sim 2 \mathrm{~W}$ while the wavelength was swept manually from blue to red. As the detuning between the pump wavelength and the active MRR's resonance became small enough to ensure sufficient modulation-instability gain in the active MRR, primary combs were initiated. As the detuning was changed further this was followed by soliton crystal microcombs. Distinctive 'palmlike' optical spectra of the soliton crystals were observed, as shown in Fig. 9 (a, b), the curtain-like spectrum a result of the interference between the tightly-packed solitons circulating around the ring [130, 131]. The soliton crystal combs featured a spacing equal to the FSR of the active MRR 48.9 GHz, enabling up to 92 channels in the C-band. Soliton crystals are coherent and low-noise and, due to the ultra-high intracavity power, could easily be generated by manually sweeping the pump wavelength — much easier than the single-soliton states.

Next, the generated soliton crystal microcombs were flattened with a Waveshaper (4000S) to equalize the power of the wavelengths. We used a feedback control path to shape the comb lines' power accurately, which were monitored by an optical spectral analyzer and compared with the desired channel weights to generate an error signal used to program the Waveshaper loss. The flattened comb spectrum is shown in Fig. 9(c). Then the 92 flattened microcomb lines were fed into a phase modulator (iXblue MPZ-LN-20, half-wave voltage $=6 \mathrm{~V}$ ) and served as optical carriers, thus broadcasting the input RF signal onto all wavelengths. The RF replicas were then spectrally sliced by the passive MRR. We employed phase modulation in combination with the notch filters (the through-port transmission of the passive MRR) to map the high-Q resonances of the passive MRR onto the RF domain, after which the input RF spectrum was channelized into $\mathrm{N}=92$ channels each centered at $f_{\mathrm{RF}}(\mathrm{k})$ with a bandwidth of $\Delta f$.

To quantify the key experimental parameters mentioned above, we measured the transmission spectrum of the passive MRR (Fig. 10(a)) using a broadband incoherent optical source using the amplified spontaneous emission of an EDFA. Both TE and TM polarized resonances were observed, as shown in the figure. As can be seen, the channelized RF frequency 

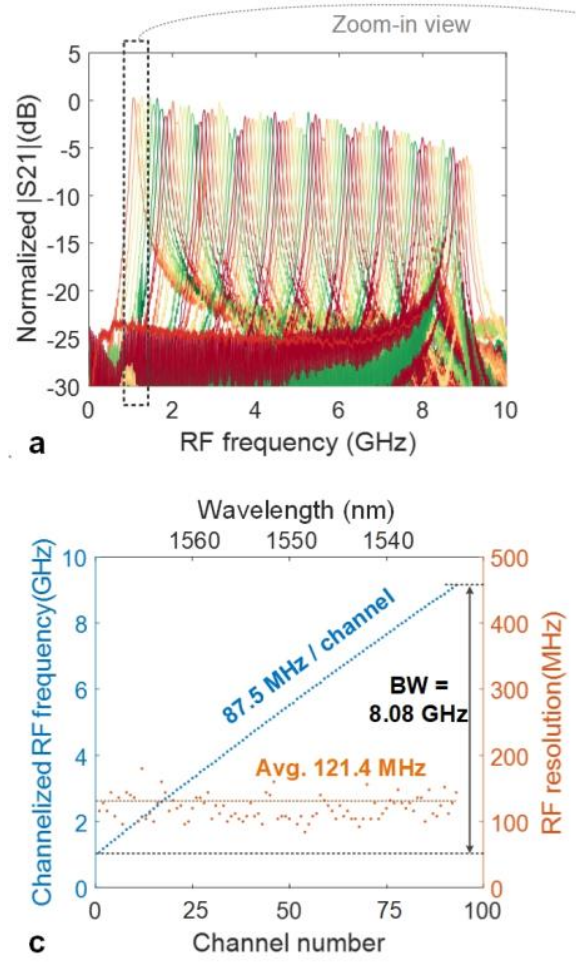

Fig. 11. Measured RF transmission spectra of (a) the 92 channels and (b) a zoom-in view of the first 4 channels. (b) Extracted channelized RF frequency and resolution. (d) Measured RF transmission spectra at different chip temperatures of the passive MRR.
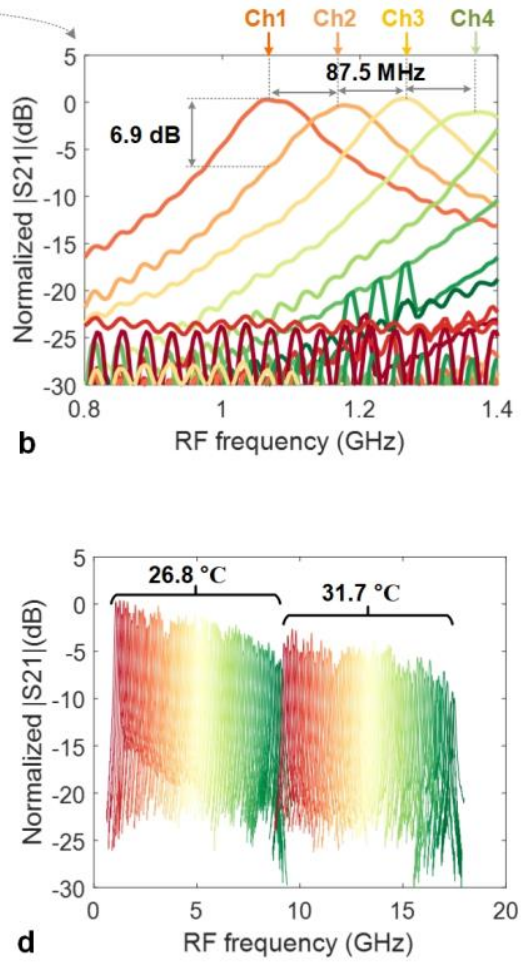

$f_{\mathrm{RF}}(\mathrm{k})$, calculated from the spacing between the comb line and the adjacent passive resonance, progressively decreases from the blue to red. The resulting RF channelized frequency (Fig. 10(b)) shows a total operation bandwidth of $8.4 \mathrm{GHz}$ and $3.8 \mathrm{GHz}$ for TE and TM resonances, respectively, with a RF channelization step of 89.5 $\mathrm{MHz}$ (TE) and $41.4 \mathrm{MHz}$ (TM) per channel. Considering the channelization resolution, denoted by the linewidth of the passive resonances, is $\sim 120 \mathrm{MHz}$ (measured in the following experiments), we employed the TE passive resonances to obtain a wider bandwidth and lower adjacent-channel interference. By aligning the polarization of the passive MRR's input light with the TE mode, the TM passive resonances were not excited and so did not affect the device performance. The birefringence and dual polarization modes of the MRRs are discussed elsewhere [114, 115].

After the passive MRR, the RF spectrum was channelized into multiple segments carried on different wavelengths which were de-multiplexed into parallel spatial paths and detected separately. We employed an RF Vector Network Analyzer to accurately measure the performance of our channelizer. The measured RF transmission spectra for 92 channels are shown in Fig. 11 (a, b), directly verifying the feasibility of our approach and the realization of 92 parallel RF channels. The imbalanced RF channel power can be equalized by including the RF transmission spectra into the comb feedback shaping loop, where the error signal is generated by the difference between the ideal channel weights with the RF power, instead of the optical power. The signal-to-noise ratio of the channelizer obtained from the RF transmission spectra was $23.7 \mathrm{~dB}$, which can be improved by increasing the extinction of the periodic optical notch filter. For the MRRs, this can be achieved by slightly adjusting the bus waveguide to MRR coupling coefficient.

The centre frequencies of each RF channel (or channelized RF frequencies), were extracted as shown in Fig. 11(c), showing a demonstrated RF channelization step of $87.5 \mathrm{MHz}$ per channel and thus a total instantaneous bandwidth of 8.08 $\mathrm{GHz}$ - matching well with our calculations from the MRR's transmission spectrum. The average RF channel resolution, calculated from the $3 \mathrm{~dB}$ bandwidth of each channel's RF transmission spectrum, was measured to be $121.4 \mathrm{MHz}$, which was enabled by the passive MRR's high Q factors. Such a high resolution greatly relaxed the requirement of the ADCs bandwidth and indicates that our approach is compatible with a wide range of digital components that typically feature relatively small bandwidths.

\section{DISCUSSION}

We note that the results for the soliton crystal-based device have a relatively high adjacent-channel crosstalk, which arises predominantly from the mismatch between the RF resolution (121.4 MHz) and the RF channelization step (87.5 MHz) which should ideally be equal. While having a channel step that is smaller than the resolution is not ideal, any resulting unwanted channel crosstalk can be discarded by eliminating redundant channels. Fine tuning the passive MRR's FSR during nanofabrication can increase the RF channelization step to match the RF resolution. This would also lead to a larger instantaneous bandwidth of $121.4 \mathrm{MHz} \times 92=11.17 \mathrm{GHz}$. Further, a high-order optical filter [181] with a flat passband and 
higher roll-off rate than the Lorentzian lineshape of the single MRR could also reduce adjacent-channel interference. Finally, a slightly higher $\mathrm{Q}$ factor ring resonator would also increase the filtering resolution.

Finally, we note that the soliton crystal channelizer's operation band is tunable over a wide range. By thermally tuning the passive MRR, the relative spacing between the source and filtering MRRs' resonances $\left(f_{\mathrm{MRR}}(1)-f_{\mathrm{OFC}}(1)\right)$ can be dynamically controlled, where a thermal response time on a millisecond time-scale is expected. As shown in Fig. 11(d), by adjusting the chip temperature of the passive MRR, we shifted the instantaneous operation band of the channelizer from 1.006-9.147 GHz to $9.227-17.49 \mathrm{GHz}$, continuously covering a total RF bandwidth of $16.48 \mathrm{GHz}$. We note that, although thermal tuning does change the passive MRR FSR, this effect was very small, in fact $\sim 3955$ times $(193.4 \mathrm{THz} / 48.9 \mathrm{GHz})$ smaller than the change in the relative spacing $\left(f_{\mathrm{MRR}}(1)-f_{\mathrm{OFC}}(1)\right)$. The change in the comb line to MRR offset (ie., $f_{\mathrm{MRR}}(1)$ $\left.f_{\mathrm{OFC}}(1)\right)$ that we induced via thermal tuning was $8.221 \mathrm{GHz}$, while the associated change in the passive MRR FSR (ie., the RF channelization step) was only $2.1 \mathrm{MHz}$, which is negligible. Therefore, this issue did not impact our device performance.

In addition, although the instantaneous RF operation band could be shifted across a wide range via thermal tuning, the lowest operation RF frequency, which was $\sim 600 \mathrm{MHz}$ in our experiments, is limited by a couple of effects. Thermal locking the center resonance wavelength of the filter to the wavelength of the optical carrier [39] places a lower limit on the RF response. Also, the relatively low spectral roll-off of the passive MRR used in our case (with a Lorentzian lineshape) also leads to a deterioration of the phase-to-intensity modulation. This can be addressed by using high-order filters with a steeper roll-off [181-188], while the thermal issues can be minimized by improving the control. The maximum operation frequency of our channelizers was determined by the Nyquist frequency of our microcomb source, which was $48.9 \mathrm{GHz} /$ $2=24.45 \mathrm{GHz}$ for the soliton crystal devices and $100 \mathrm{GHz}$ for the $200 \mathrm{GHz}$ microcomb device. Hence, the $200 \mathrm{GHz}$ system had a much higher potential maximum RF operation frequency of $100 \mathrm{GHz}$ - although this comes with a tradeoff with the number of comb lines within the available wavelength range.

\section{CONCLUSIONS}

We demonstrate broadband RF channelizers based on CMOS-compatible integrated optical frequency comb sources. Broadband $200 \mathrm{GHz}$-spaced Kerr combs as well as soliton crystal combs with a 49GHz spacing are employed. Both combs provided a record large number of wavelength channels as well as a large RF operation bandwidth. The $200 \mathrm{GHz}$ combbased device has a large potential upper bandwidth of $100 \mathrm{GHz}$, while the soliton crystal device, due to its small $(49 \mathrm{GHz})$ spacing that and closely matches the FSRs of the passive MRR filter, up to 92 available wavelength channels were generated, resulting in a broad RF instantaneous bandwidth of $8.08 \mathrm{GHz}$. A high RF slicing resolution of $121.4 \mathrm{MHz}$ was achieved by the high-Q passive MRR that served as the periodic optical filter for spectra slicing for both devices. For the soliton crystal device, phase to intensity modulation format conversion was employed to ensure stable signal detection without the need for any external local oscillator paths. Dynamic tuning of the RF operation frequency range was achieved through for both devices through thermal control applied to the passive MRR, achieving RF operation up to $17.49 \mathrm{GHz}$ for the soliton crystal device. This device also achieved broadband channelization of RF frequencies from $1.7 \mathrm{GHz}$ to 19 $\mathrm{GHz}$ with a high spectral slice resolution of $1 \mathrm{GHz}$ via thermal tuning of a passive on-chip MRR with a $\mathrm{Q}$ factor of $1.549 \times 10^{6}$. These micro-comb based RF channelizers are highly attractive for achieving broadband RF channelization with large channel numbers, high resolution, small footprint, and potentially low cost. They feature massively parallel channels and are highly promising for broadband instantaneous signal detection and processing, representing a solid step towards fully integrated photonic receivers for modern RF systems.

\section{References}

[1] S. Radic, and D. J. Moss, and B. J. Eggleton, "Nonlinear optics in communications: From crippling impairment to ultrafast tools", Chapter 20, p759-828 in Optical Fiber Telecommunications V: Components and Sub-systems, Ed. Ivan P. Kaminow, Tingye Li, and Alan E. Willner, Academic Press, Oxford, UK, (2008).

[2] J. Leuthold, C. Koos, and W. Freude, "Nonlinear silicon photonics," Nat. Photon., vol. 4, no. 8, 535-544 (2010).

[3] L. Li, et al. "All-optical regenerator of multi-channel signals," Nature Communications, vol. 8, Article: 884 (2017).

[4] F. Li, et al., "All-optical XOR logic gate for 40Gb/s DPSK signals via FWM in a silicon nanowire," Optics Express, vol. 19, no. 21, 20364-20371 (2011). DOI: 10.1364/OE.19.020364.

[5] F. Li, et al., "Error-free All-Optical Demultiplexing at 160Gb/s via FWM in a Silicon Nanowire," Optics Express, vol. 18, no. 4, pp. 3905-3910 (2010). DOI: 10.1364/OE.18.003905.

[6] H. Ji, et al., "1.28-Tb/s Demultiplexing of an OTDM DPSK Data Signal Using a Silicon Waveguide," Photonics Technology Letters, vol. 22, no. 23, 1762-1764 (2010).

[7] C. Monat, et al., "Investigation of phase matching for third-harmonic generation in silicon slow light photonic crystal waveguides using Fourier optics," Optics Express, vol. 18, no. 7, pp. 6831-6840 (2010). DOI: 10.1364/OE.18.006831. 
[8] B. Corcoran, et al., "Optical Signal Processing on a Silicon Chip at 640Gb/s Using Slow-Light," Optics Express, vol. 18, no. 8, pp. 7770-7781 (2010). DOI: 10.1364/OE.18.007770.

[9] V. G. Ta'eed, et al., "Integrated all-optical pulse regenerator in chalcogenide waveguides," Optics Letters, vol. 30, no. 21, pp. 29002902 (2005).

[10]M. Rochette, et al., "Bit-error-ratio improvement with 2R optical regenerators," IEEE Photonics Technology Letters, vol. 17, no. 4, pp. 908-910 (2005).

[11]M. Ferrera, et al., "CMOS compatible integrated all-optical radio frequency spectrum analyzer," Optics Express, vol. 22, no. 18, pp. 21488 - 21498 (2014). DOI: 10.1364/OE.22.021488.

[12]C. Monat, et al., "Integrated optical auto-correlator based on third-harmonic generation in a silicon photonic crystal waveguide," Nature Communications, vol. 5, Article:3246 (2014). doi:10.1038/ncomms4246.

[13]F. Li, et al., "All-optical wavelength conversion for $10 \mathrm{~Gb} / \mathrm{s}$ DPSK signals in a silicon ring resonator," Optics Express, vol. 19, no. 23, pp. 22410-22416 (2011)

[14]T. D. Vo, et al., "Silicon-Chip-Based Real-Time Dispersion Monitoring for 640 Gbit/s DPSK Signals," IEEE Journal of Lightwave Technology, vol. 29, no. 12, pp. 1790-1796 (2011).

[15]M. Ferrera, et al., "All-optical $1^{\text {st }}$ and $2^{\text {nd }}$ order integration on a chip," Optics Express, vol. 19, no. 23, pp. $23153-23161,2011$.

[16]B. Corcoran, et al., "Silicon nanowire based radio-frequency spectrum analyzer," Optics Express, vol. 18, no. 19, pp. 20190-20200 (2010). DOI: 10.1364/OE.18.020190.

[17]B. Corcoran, et al., "Green light emission in silicon through slow-light enhanced third-harmonic generation in photonic-crystal waveguides," Nature Photonics, vol. 3, no. 4, pp. 206-210 (2009). doi:10.1038/nphoton.2009.28.

[18]D. J. Moss, H. M. van Driel, and J. E. Sipe, "Dispersion in the anisotropy of optical third-harmonic generation in silicon," Opt. Lett., vol. 14, no. 1, pp. 57-59 (1989).

[19]J. E. Sipe, D. J. Moss, and H. M. van Driel, "Phenomenological Theory of Optical Second- And Third-Harmonic Generation Form Cubic Centrosymmetric Crystals," Phys. Rev. B, vol. 35, no. 3, pp. 1129-1141 (1987).

[20]D. J. Moss, E. Ghahramani, J. E. Sipe, and H. M. van Driel, "Band-structure calculation of dispersion and anisotropy in $\chi \rightarrow(3)$ for third-harmonic generation in Si, Ge, and GaAs," Phys. Rev. B, vol. 41, no. 3, pp. 1542-1560 (1990).

[21]D. J. Moss, H. M. van Driel, and J. E. Sipe, "Third harmonic generation as a structural diagnostic of ion implanted amorphous and crystalline silicon," Appl. Phy. Lett., vol. 48, no. 17, pp. 1150 (1986).

[22]D. J. Moss, et al., "Ultrafast all-optical modulation via two-photon absorption in silicon-insulator waveguides," Electronics Letters, vol. 41, no. 6, pp. 320-321 (2005). DOI:10.1049/el:20058051

[23]M. R. E. Lamont, et al.,"Two-photon absorption effects on self-phase-modulation-based 2R optical regeneration," Photonics Technology Letters, vol. 18, no. 10, pp. 1185-1187 (2006). DOI:10.1109/LPT.2006.874718.

[24]A.Tuniz, G. Brawley, D. J. Moss, and B. J. Eggleton, "Two-photon absorption effects on Raman gain in single mode As2Se3 chalcogenide glass fiber," Optics Express, vol. 16, no. 22, pp. 18524-18534 (2008). DOI: 10.1364/OE.16.018524.

[25]M.D. Pelusi, F. Luan, E. Magi, M.R.E. Lamont, D. J. Moss, B.J. Eggleton, J.S. Sanghera, L. B. Shaw, and I.D. Aggarwal, "High bit rate all-optical signal processing in a fiber photonic wire", Optics Express vol.16, 11506-11512 (2008).

[26]M. Lee, et al., "Photosensitive post tuning of chalcogenide photonic crystal waveguides," Optics Express, vol. 15, no. 3, pp. 12771285 (2007). DOI:10.1364/OE.15.001277

[27]S. Tomljenovic-Hanic, M. J. Steel, C. M. d. Sterke and D. J. Moss, "High-Q cavities in photosensitive photonic crystals", Optics Letters, 32, no. 5, pp. 542-544 (2007).

[28]C. Grillet, et al., "Nanowire coupling to photonic crystal nanocavities for single photon sources," Optics Express, vol. 15, no. 3, pp. 1267-1276 (2007). DOI:10.1364/OE.15.001267

[29]V. G. Ta'eed, et al., "Ultrafast all-optical chalcogenide glass photonic circuits", Optics Express, vol. 15, no. 15, pp. $9205-9221$ (2007).

[30]D. Freeman, et al., "Chalcogenide Glass Photonic Crystal Devices", Photonic and Electromagnetic Crystal Structures, Photonics and Nanostructures-Fundamentals and Applications, Science Direct Elsevier Publishing, vol. 6, no. 1, pp. 3-11 (2008). doi:10.1016/j.photonics.2007.11.001.

[31]C. Grillet, et al., "Characterization and modeling of Fano resonances in chalcogenide photonic crystal membranes", Optics Express, vol. 14, no. 1, pp. 369-376 (2006).

[32]V. G. Ta'eed, et al., "Self-phase modulation based integrated optical regeneration in chalcogenide waveguides", IEEE Journal of Selected Topics in Quantum Electronics, vol. 12, no. 3, pp. 360-370 (2006).

[33]M. Shokooh-Saremi, et al., "High performance Bragg gratings in chalcogenide rib waveguides written with a modified Sagnac interferometer: experiment and modeling", Journal of the Optical Society of America B (JOSA B), vol. 23, no. 7, pp. 1323-1331 (2006).

[34]M. R. E. Lamont, et al., "Error-free wavelength conversion via cross phase modulation in $5 \mathrm{~cm}$ of As2 $\mathrm{S}_{3}$ chalcogenide glass rib waveguide", Electronics Letters, vol. 43, pp. 945-947 (2007).

[35]K. Ikeda, R. E. Saperstein, N. Alic, and Y. Fainman, "Thermal and Kerr nonlinear properties of plasma-deposited silicon nitride/silicon dioxide waveguides", Opt. Express, vol. 16, pp. 12987-12994 (2008).

[36] J. S. Levy, A. Gondarenko, M. A. Foster, et al., "CMOS-compatible multiple-wavelength oscillator for on-chip optical interconnects," Nature Photonics, vol. 4, 1, pp. 37-40 (2010).

[37] L. Razzari, D. Duchesne, M. Ferrera, et al., "CMOS-compatible integrated optical hyper-parametric oscillator," Nature Photonics, vol. 4, no. 1, pp. 41-45 (2010). 
[38] D. J. Moss, R. Morandotti, A. L. Gaeta, et al., "New CMOS-compatible platforms based on silicon nitride and Hydex for nonlinear optics," Nature Photonics, vol. 7, no. 8, pp. 597-607 (2013).

[39] M. Ferrera, L. Razzari, D. Duchesne, et al., "Low-power continuous-wave nonlinear optics in doped silica glass integrated waveguide structures," Nature Photonics, vol. 2, no. 12, pp. 737-740 (2008).

[40] A. Pasquazi, et al., "Sub-picosecond phase-sensitive optical pulse characterization on a chip", Nature Photonics, vol. 5, no. 10, pp. 618-623 (2011). DOI: 10.1038/nphoton.2011.199.

[41] D. Duchesne, M. Peccianti, M. R. E. Lamont, et al., "Supercontinuum generation in a high index doped silica glass spiral waveguide," Optics Express, vol. 18, no, 2, pp. 923-930 (2010).

[42] M. Ferrera, et al., "On-chip CMOS-compatible all-optical integrator", Nature Communications, vol. 1, Article 29 (2010).

[43] A. Pasquazi, et al., "All-optical wavelength conversion in an integrated ring resonator," Optics Express, vol. 18, no. 4, pp. 38583863 (2010).

[44] A. Pasquazi, Y. Park, J. Azana, et al., "Efficient wavelength conversion and net parametric gain via Four Wave Mixing in a high index doped silica waveguide," Optics Express, vol. 18, no. 8, pp. 7634-7641 (2010).

[45] M. Peccianti, M. Ferrera, L. Razzari, et al., "Subpicosecond optical pulse compression via an integrated nonlinear chirper," Optics Express, vol. 18, no. 8, pp. 7625-7633 (2010).

[46] D. Duchesne, M. Ferrera, L. Razzari, et al., "Efficient self-phase modulation in low loss, high index doped silica glass integrated waveguides," Optics Express, vol. 17, no. 3, pp. 1865-1870 (2009).

[47] A. Pasquazi, M. Peccianti, L. Razzari, D. J. Moss, S. Coen, M. Erkintalo, Y. K. Chembo, T. Hansson, S. Wabnitz, P. Del'Haye, X. X. Xue, A. M. Weiner, and R. Morandotti, "Micro-combs: A novel generation of optical sources," Physics Reports, vol. 729, pp. 181 (2018).

[48] P. Del'Haye, A. Schliesser, O. Arcizet, T. Wilken, R. Holzwarth, and T. J. Kippenberg, "Optical frequency comb generation from a monolithic microresonator", Nature, vol. 450, pp. 1214-1217 (2007).

[49] M. Peccianti, et al., "Demonstration of an ultrafast nonlinear microcavity modelocked laser", Nature Communications, vol. 3, pp. 765 (2012).

[50] M. Kues, et al., "Passively modelocked laser with an ultra-narrow spectral width", Nature Photonics, vol. 11, no. 3, pp. 159 (2017) DOI:10.1038/nphoton.2016.271

[51] A. Pasquazi, L. Caspani, M. Peccianti, et al., "Self-locked optical parametric oscillation in a CMOS compatible microring resonator: a route to robust optical frequency comb generation on a chip," Optics Express, vol. 21, no. 11, pp. 13333-13341 (2013).

[52] A. Pasquazi, M. Peccianti, B. E. Little, et al., "Stable, dual mode, high repetition rate mode-locked laser based on a microring resonator," Optics Express, vol. 20, no. 24, pp. 27355-27362 (2012).

[53] C. Reimer, L. Caspani, M. Clerici, et al., "Integrated frequency comb source of heralded single photons," Optics Express, vol. 22, no. 6, pp. 6535-6546 (2014).

[54] C. Reimer, et al., "Cross-polarized photon-pair generation and bi-chromatically pumped optical parametric oscillation on a chip", Nature Communications, vol. 6, Article 8236 (2015). DOI: 10.1038/ncomms9236

[55] L. Caspani, C. Reimer, M. Kues, et al., "Multifrequency sources of quantum correlated photon pairs on-chip: a path toward integrated Quantum Frequency Combs," Nanophotonics, vol. 5, no. 2, pp. 351-362 (2016).

[56] C. Reimer, M. Kues, P. Roztocki, B. Wetzel, F. Grazioso, B. E. Little, S. T. Chu, T. Johnston, Y. Bromberg, L. Caspani, D. J. Moss, and R. Morandotti, "Generation of multiphoton entangled quantum states by means of integrated frequency combs," Science, vol. 351, no. 6278, pp. 1176-1180 (2016).

[57] M. Kues, et al., "On-chip generation of high-dimensional entangled quantum states and their coherent control", Nature, vol. 546, no. 7660, pp. 622-626 (2017).

[58] P. Roztocki, M. Kues, C. Reimer, B. Wetzel, S. Sciara, Y. Zhang, A. Cino, B. E. Little, S. T. Chu, D. J. Moss, and R. Morandotti, "Practical system for the generation of pulsed quantum frequency combs," Optics Express, vol. 25, no. 16, pp. 18940-18949 (2017).

[59] Y. Zhang, et al., "Induced photon correlations through superposition of two four-wave mixing processes in integrated cavities", Laser and Photonics Reviews, vol. 14, no. 7, 2000128 (2020). DOI: 10.1002/lpor.202000128

[60] M. Kues, C. Reimer, A. Weiner, J. Lukens, W. Munro, D. J. Moss, and R. Morandotti, "Quantum Optical Micro-combs", Nature Photonics, vol. 13, no.3, pp. 170-179 (2019).

[61] C. Reimer, et al.,"High-dimensional one-way quantum processing implemented on d-level cluster states", Nature Physics, vol. 15 , no.2, pp. 148-153 (2019).

[62] P. Marin-Palomo, et al., "Microresonator-based solitons for massively parallel coherent optical communications", Nature, vol. 546, no. 7657, pp. 274 (2017)

[63] J. Pfeifle, V. Brasch, M. Lauermann, Y. Yu, D. Wegner, T. Herr, K. Hartinger, et al., "Coherent terabit communications with microresonator Kerr frequency combs", Nature Photonics, vol. 8, no. 5, pp. 375-380, 2014.

[64] B. Corcoran, et al., "Ultra-dense optical data transmission over standard fiber with a single chip source", Nature Communications, vol. 11, Article:2568 (2020). DOI:10.1038/s41467-020-16265-x.

[65] X. Xu, et al., "Photonic perceptron based on a Kerr microcomb for scalable high speed optical neural networks", Laser and Photonics Reviews, vol. 14, no. 8, 2000070 (2020). DOI:10.1002/lpor.202000070.

[66] X. Xu, et al., "11 TeraFLOPs photonic convolutional accelerator for deep learning convolutional optical neural networks", Nature, vol. 589 (7840), 44-51 (2021).

[67] J. Feldmann, et al., "Parallel convolution processing using an integrated photonic tensor core", arXiv:2002.00281, 2020. 
[68] D. T. Spencer, et al., “An optical-frequency synthesizer using integrated photonics”, Nature, vol. 557, no. 7703, pp. 81-85, 2018.

[69] T. J. Kippenberg, A. L. Gaeta, M. Lipson, and M. L. Gorodetsky, "Dissipative Kerr solitons in optical microresonators," Science, vol. 361, no. 6402, 2018.

[70] A. L. Gaeta, M. Lipson, and T. J. Kippenberg, "Photonic-chip-based frequency combs," Nature Photonics, vol. 13, no. 3, pp. 158169, Mar. 2019.

[71] P. Del'Haye, T. Herr, E. Gavartin, M. L. Gorodetsky, R. Holzwarth, and T. J. Kippenberg, "Octave spanning tunable frequency comb from a microresonator", Physical Review Letters, vol. 107, no. 6, pp. 063901, 2011.

[72] T. J. Kippenberg, R. Holzwarth, S. A. Diddams, "Microresonator-based optical frequency combs", Science, vol. 332, no. 6029, pp. 555-559, 2011.

[73] T. Herr, V. Brasch, J. D. Jost, C. Y. Wang, N. M. Kondratiev, M. L. Gorodetsky, et al., "Temporal solitons in optical microresonators", Nature Photonics, vol. 8, no. 2, pp. 145-152, 2014.

[74] F. Ferdous, H. Miao, D. E. Leaird, K. Srinivasan, J. Wang, L. Chen, L. T. Varghese, and A. M. Weiner, "Spectral line-by-line pulse shaping of on-chip microresonator frequency combs", Nature Photonics, vol. 5, no. 12, pp. 770, 2011.

[75] X. Xue, P. H. Wang, Y. Xuan, M. Qi, and A. M. Weiner, "Microresonator Kerr frequency combs with high conversion efficiency", Laser \& Photonics Reviews, vol. 11, no. 1, 2017.

[76] X. Xue, M. Qi, and A. M. Weiner, "Normal-dispersion microresonator Kerr frequency combs", Nanophotonics, vol. 5, no. 2, pp. 244-262, 2016.

[77] C. Grillet, et al., "Amorphous Silicon Nanowires with Record High Nonlinearity, FOM, and Optical Stability”, Optics Express, vol. 20, no. 20. pp. 22609-22615 (2012).

[78] J. W. Choi, B. Sohn, G. F. R. Chen, D. K. T. Ng, and D. T. H. Tan, "Soliton-effect optical pulse compression in CMOS-compatible ultra-silicon-rich nitride waveguides," APL Photonics, HIBSP2019, pp. 110804 (2020).

[79] J. Capmany, and D. Novak, "Microwave photonics combines two worlds," Nat. Photonics, vol. 1, no. 6, pp. 319-330, 2007.

[80] J. P. Yao, "Microwave photonics," Journal of Lightwave Technol., vol. 27, no. 1-4, pp. 314-335, Jan-Feb. 2009.

[81] D. Marpaung, J. Yao, and J. Capmany, "Integrated microwave photonics," Nature Photonics, vol. 13, no. 2, pp. 80-90, Feb. 2019.

[82] J. Azaña, "Ultrafast analog all-optical signal processors based on fiber-grating devices," IEEE Photonics Journal, vol. 2, no. 3, 359386. 2010.

[83] J.Capmany, B.Ortega, and D. Pastor, "A tutorial on microwave photonic filters," Journal of Lightwave Technol., vol. 24, no. 1, pp.201-229, 2006.

[84] V. R. Supradeepa et al., "Comb-based radiofrequency photonic filters with rapid tunability and high selectivity," Nat. Photonics, vol. 6, no. 3, pp. 186-194, Mar.2012.

[85] J. Wu, X. Xu, T. G. Nguyen, S. T. Chu, B. E. Little, R. Morandotti, A. Mitchell, and D. J. Moss, "RF Photonics: An Optical Microcombs' Perspective," IEEE J. Sel. Top. Quantum Electron., vol. 24, no. 4, 6101020 (2018). DOI: 10.1109/JSTQE.2018.2805814.

[86] V. Torres-Company, and A. M. Weiner, "Optical frequency comb technology for ultra-broadband radio-frequency photonics," Laser Photonics Rev, vol. 8, no. 3, pp. 368-393, May. 2014.

[87] Z. Jiang, C. B. Huang, D. E. Leaird, and A. M. Weiner, "Optical arbitrary waveform processing of more than 100 spectral comb lines," Nat Photonics, vol. 1, no. 8, pp. 463-467, Aug. 2007.

[88] Y. Liu, J. Hotten, A. Choudhary, B. J. Eggleton, and D. Marpaung, "All-optimized integrated RF photonic notch filter," Opt. Lett., vol. 42, no. 22, pp. 4631-4634, Nov 15. 2017.

[89] Y. Liu, D. Marpaung, A. Choudhary, J. Hotten, and B. J. Eggleton, "Link performance optimization of chip-based Si3N4 microwave photonic filters," J. Lightwave Technol., vol. 36, no. 19, pp. 4361-4370, 2018.

[90] Y. Liu, Y. Yu, S. X. Yuan, X. B. Xu, and X. L. Zhang, "Tunable megahertz bandwidth microwave photonic notch filter based on a silica microsphere cavity," Opt. Lett., vol. 41, no. 21, pp. 5078-5081, Nov 1. 2016.

[91] D. Marpaung, B. Morrison, M. Pagani, R. Pant, D. Y. Choi, B. Luther-Davies, S. J. Madden, and B. J. Eggleton, "Low-power, chipbased stimulated Brillouin scattering microwave photonic filter with ultrahigh selectivity," Optica, vol. 2, no. 2, 76-83 (2015).

[92] A. Choudhary, B. Morrison, I. Aryanfar, S. Shahnia, M. Pagani, Y. Liu, K. Vu, S. Madden, D. Marpaung, and B. J. Eggleton, "Advanced integrated microwave signal processing with giant on-chip Brillouin gain," J. Lightwave Technol., vol. 35, no. 4, pp. 846854, Feb 15. 2017.

[93] D. Marpaung, B. Morrison, R. Pant, and B. J. Eggleton, "Frequency agile microwave photonic notch filter with anomalously high stopband rejection," Opt. Lett., vol. 38, no. 21, pp. 4300-4303, Nov 1. 2013.

[94] X. Q. Zhu, F. Y. Chen, H. F. Peng, and Z. Y. Chen, "Novel programmable microwave photonic filter with arbitrary filtering shape and linear phase," Opt. Express, vol. 25, no. 8, pp. 9232-9243, Apr 17. 2017.

[95] F. Jiang, Y. Yu, H. T. Tang, L. Xu, and X. L. Zhang, "Tunable bandpass microwave photonic filter with ultrahigh stopband attenuation and skirt selectivity," Opt. Express, vol. 24, no. 16, pp. 18655-18663, Aug 8. 2016.

[96] Z. J. Zhu, H. Chi, T. Jin, S. L. Zheng, X. F. Jin, and X. M. Zhang, "All-positive-coefficient microwave photonic filter with rectangular response," Opt. Lett., vol. 42, no. 15, pp. 3012-3015, Aug 1. 2017.

[97] G. Yu, W. Zhang, and J. A. R. Williams, "High-performance microwave transversal filter using fiber Bragg grating arrays," IEEE Photonic Tech L, vol. 12, no. 9, pp. 1183-1185, Sep. 2000.

[98] J. S. Leng, W. Zhang, and J. A. R. Williams, "Optimization of superstructured fiber Bragg gratings for microwave photonic filters response," IEEE Photonic Tech L, vol. 16, no. 7, pp. 1736-1738, Jul. 2004. 
[99] D. B. Hunter, R. A. Minasian, and P. A. Krug, "Tunable optical transversal filter based on chirped gratings," Electron. Lett., vol. 31, no. 25, pp.2205-2207, Dec 7. 1995.

[100] E. Hamidi, D. E. Leaird, and A. M. Weiner, "Tunable Programmable Microwave Photonic Filters Based on an Optical Frequency Comb,” IEEE Journal of Microwave Theory, vol. 58, no. 11, pp. 3269-3278, Nov. 2010.

[101] R. Wu et al., "Generation of very flat optical frequency combs from continuous-wave lasers using cascaded intensity and phase modulators driven by tailored radio frequency waveforms," Opt. Lett., vol. 35, no. 19, pp. 3234-3236, 2010.

[102] S. Mansoori, and A. Mitchell, "RF transversal filter using an AOTF," IEEE Photonic Tech L, vol. 16, no. 3, pp. 879-881, 2004.

[103] M. Delgado-Pinar, J. Mora, A. Diez, M. V. Andres, B. Ortega, and J. Capmany, "Tunable and reconfigurable microwave filter by use of a Bragg-grating-based acousto-optic superlattice modulator," Opt. Lett., vol. 30, no. 1, pp. 8-10, Jan 1. 2005.

[104] W. Z. Li, and J. P. Yao, "Optical frequency comb generation based on repeated frequency shifting using two Mach-Zehnder modulators and an asymmetric Mach-Zehnder interferometer," Opt. Express, vol. 17, no. 26, pp. 23712-23718, Dec 21. 2009.

[105] C. H. Chen, C. He, D. Zhu, R. H. Guo, F. Z. Zhang, and S. L. Pan, "Generation of a flat optical frequency comb based on a cascaded polarization modulator and phase modulator," Opt. Lett., vol. 38, no. 16, pp. 3137-3139, Aug 15. 2013.

[106] T. Saitoh, M. Kourogi, and M. Ohtsu, "An optical frequency synthesizer using a waveguide-type optical frequency comb generator at 1.5-mu m wavelength," IEEE Photonic Tech L, vol. 8, no. 11, pp. 1543-1545, Nov. 1996.

[107] T. G. Nguyen et al., "Integrated frequency comb source-based Hilbert transformer for wideband microwave photonic phase analysis," Opt. Express, vol. 23, no. 17, pp. 22087-22097 (2015).

[108] X. Xue, et al., "Programmable single-bandpass photonic RF filter based on a Kerr comb from a microring," Journal of Lightwave Technol., vol. 32, no. 20, pp. 3557-3565, Oct. 2014.

[109] X. Xu, J. Wu, M. Shoeiby, T. G. Nguyen, S. T. Chu, B. E. Little, R. Morandotti, A. Mitchell, and D. J. Moss, "Reconfigurable broadband microwave photonic intensity differentiator based on an integrated optical frequency comb source," APL Photonics, vol. 2, no. 9, 096104 (2017).

[110] X. Xu, M. Tan, J. Wu, R. Morandotti, A. Mitchell, and D. J. Moss, "Microcomb-based photonic RF signal processing", IEEE Photonics Technology Letters, vol. 31 no. 23 1854-1857 (2019).

[111] X. Xu, et al., "Advanced RF and microwave functions based on an integrated optical frequency comb source," Opt. Express, vol. 26, no. 3, pp. 2569-2583 (2018).

[112] X. Xue, et al., "Microcomb-based true-time-delay network for microwave beamforming with arbitrary beam pattern control," Journal of Lightwave Technology, vol. 36, no. 12, pp. 2312-2321, Jun. 2018.

[113] X. Xu, et al., "Broadband RF channelizer based on an integrated optical frequency Kerr comb source," Journal of Lightwave Technology, vol. 36, no. 19, pp. 4519-4526 (2018).

[114] X. Xu, et al., "Continuously tunable orthogonally polarized RF optical single sideband generator based on micro-ring resonators," Journal of Optics, vol. 20, no. 11, 115701 (2018).

[115] X. Xu, et al., "Orthogonally polarized RF optical single sideband generation and dual-channel equalization based on an integrated microring resonator," Journal of Lightwave Technology, vol. 36, no. 20, pp. 4808-4818 (2018).

[116] X. Xu, et al., "Photonic microwave true time delays for phased array antennas using a $49 \mathrm{GHz}$ FSR integrated optical micro-comb source," Photonics Res, vol. 6, no. 5, pp. B30-B36 (2018).

[117] X. Xu, et al., "Advanced adaptive photonic RF filters with 80 taps based on an integrated optical micro-comb source," Journal of Lightwave Technology, vol. 37, no. 4, pp. 1288-1295 (2019).

[118] W. Liang, et al., "High spectral purity Kerr frequency comb radio frequency photonic oscillator," Nature Communications, vol. 6 pp. 7957. 2015.

[119] J. Liu, et al., "Photonic microwave generation in the X-and K-band using integrated soliton microcombs" Nature Photonics, vol. $14,1-6,2020$.

[120] X. Xu et al., Broadband microwave frequency conversion based on an integrated optical micro-comb source", Journal of Lightwave Technology, vol. 38 no. 2, pp. 332-338 (2020).

[121] M. Tan et al., "Photonic RF and microwave filters based on $49 \mathrm{GHz}$ and 200GHz Kerr microcombs", Optics Comm. vol. 465 , Article: 125563, Feb. 22 (2020).

[122] X. Xu et al., "Broadband photonic RF channelizer with 90 channels based on a soliton crystal microcomb", Journal of Lightwave Technology, Vol. 38, no. 18, pp.5116 - 5121 (2020). doi: 10.1109/JLT.2020.2997699.

[123] X. Xu et al., "Photonic RF and microwave integrator with soliton crystal microcombs", IEEE Transactions on Circuits and Systems II: Express Briefs, Vol. 67 (12) 3582-3586 (2020). DOI:10.1109/TCSII.2020.2995682.

[124] X. Xu et al., "Photonic RF phase-encoded signal generation with a microcomb source", Journal of Lightwave Technology, vol. 38, no. 7, pp. 1722-1727 (2020).

[125] X. Xu et al., "High performance RF filters via bandwidth scaling with Kerr micro-combs," APL Photonics, vol. 4, no. 2, pp. $026102(2019)$.

[126] M. Tan et al., "Microwave and RF photonic fractional Hilbert transformer based on a $50 \mathrm{GHz}$ Kerr micro-comb", Journal of Lightwave Technology, vol. 37, no. 24, pp. 6097 - 6104 (2019).

[127] M. Tan et al., "RF and microwave fractional differentiator based on photonics", IEEE Transactions on Circuits and Systems: Express Briefs, Vol. 67, No. 11, pp. 2767 - 2771 (2020). DOI:10.1109/TCSII.2020.2965158.

[128] M. Tan et al., "Photonic RF arbitrary waveform generator based on a soliton crystal micro-comb source", Journal of Lightwave Technology, Vol. 38, No. 22, pp. 6221-6226, Oct 22 (2020). DOI: 10.1109/JLT.2020.3009655. 
[129] M. Tan, X. Xu, J. Wu, R. Morandotti, A. Mitchell, and D. J. Moss, "RF and microwave high bandwidth signal processing based on Kerr Micro-combs", Advances in Physics X, VOL. 6, NO. 1, 1838946 (2020). DOI:10.1080/23746149.2020.1838946.

[130] D. C. Cole, E. S. Lamb, P. Del'Haye, S. A. Diddams, and S. B. Papp, "Soliton crystals in Kerr resonators," Nat. Photonics, vol. 11, no. 10, pp. 671-676, Oct. 2017.

[131] W. Wang, Z. Lu, W. Zhang, S. T. Chu, B. E. Little, L. Wang, X. Xie, M. Liu, Q. Yang, L. Wang, J. Zhao, G. Wang, Q. Sun, Y. Liu, Y. Wang, and W. Zhao, "Robust soliton crystals in a thermally controlled microresonator," Opt. Lett., vol. 43, no. 9, pp. 20022005, 2018.

[132] B. Stern, X. Ji, Y. Okawachi, A. L. Gaeta, and M. Lipson, "Battery-operated integrated frequency comb generator”, Nature, vol. 562, no. 7727, pp. 401, 2018.

[133] X. Xue, et al., "Mode-locked dark pulse Kerr combs in normal-dispersion microresonators," Nature Phot., vol. 9 (9) 594. (2015).

[134] H. Bao et al., "Laser cavity-soliton microcombs," Nature Photonics, vol. 13, no. 6, pp. 384-389 (2019).

[135] X. Xue, X. Zheng, and B. Zhou, "Super-efficient temporal solitons in mutually coupled optical cavities," Nature Photonics, May 2019.

[136] H. Zhou, et al., "Soliton bursts and deterministic dissipative Kerr soliton generation in auxiliary-assisted microcavities," Light: Science \& Applications, vol. 8, no. 1, pp. 50, 2019.

[137] H. Bao et al., "Turing patterns in a fibre laser with a nested micro-resonator: robust and controllable micro-comb generation", Physical Review Research, vol. 2, pp. 023395 (2020).

[138] L. D. Lauro, J. Li, D. J. Moss, R. Morandotti, S. T. Chu, M. Peccianti, and A. Pasquazi, "Parametric control of thermal selfpulsation in micro-cavities," Opt. Lett. vol. 42, no. 17, pp. 3407-3410, Aug. 2017.

[139] H. Bao et al., "Type-II micro-comb generation in a filter-driven four wave mixing laser," Photonics Research, vol. 6, no. 5, pp. B67-B73 (2018).

[140] B. Shen, Chang, L., Liu, J., et al., "Integrated turnkey soliton microcombs," Nature, vol. 582, pp. 365-369, 2020.

[141] J. Capmany and D. Novak, "Microwave photonics combines two worlds," Nat. Photonics, vol. 1, no. 6, pp. 319-330, Jun. 2007.

[142] J. P. Yao, "Microwave Photonics," J. Lightwave Technol., vol. 27, no. 1-4, pp. 314-335, Jan. 2009.

[143] S. L. Pan and J. P. Yao, "Photonics-Based Broadband Microwave Measurement," J. Lightwave Technol., vol. 35, no. 16, pp. 34983513, 2017.

[144] J. Azana, C. Madsen, K. Takiguchi, and G. Cincotti, "Guest editorial - Optical signal processing,” J. Lightwave Technol., vol. 24, no. 7, pp. 2484-2486, Jul. 2006.

[145] D. Marpaung et al, "Nonlinear Integrated Microwave Photonics," J. Lightwave Technol., vol. 32, no. 20, pp. 3421-3427, Oct. 2014.

[146] R. A. Minasian, "Ultra-Wideband and Adaptive Photonic Signal Processing of Microwave Signals," IEEE J. Quantum Elect., vol. 52, no. $1,2016$.

[147] X. H. Zou, B. Lu, W. Pan, L. S. Yan, A. Stohr, and J. P. Yao, "Photonics for microwave measurements," Laser Photonics Rev., vol 10, no. 5, pp. 711-734, Sep. 2016.

[148] K. Xu, R. X. Wang, Y. T. Dai, F. F. Yin, J. Q. Li, Y. F. Ji, and J. T. Lin, "Microwave photonics: radio-over-fiber links, systems, and applications," Photonics Res., vol. 2, no. 4, pp. B54-B63, Aug. 2014.

[149] S. L. Pan, D. Zhu, S. F. Liu, K. Xu, Y. T. Dai, T. L. Wang, J. G. Liu, N. H. Zhu, Y. Xue, and N. J. Liu, "Satellite Payloads Pay Off," IEEE Microw. Mag., vol. 16, no. 8, pp. 61-73, Sep. 2015.

[150] H. W. Chen, R. Y. Li, C. Lei, Y. Yu, M. H. Chen, S. G. Yang, and S. Z. Xie, "Photonics-Assisted Serial Channelized RadioFrequency Measurement System With Nyquist-Bandwidth Detection," IEEE Photonics J., vol. 6, no. 6, Dec. 2014.

[151] X. J. Xie, Y. T. Dai, Y. Ji, K. Xu, Y. Li, J. Wu, and J. T. Lin, "Broadband Photonic Radio-Frequency Channelization Based on a 39-GHz Optical Frequency Comb,” IEEE Photonic Tech. L., vol. 24, no. 8, pp. 661-663, Apr. 2012.

[152] W. S. Wang, R. L. Davis, T. J. Jung, R. Lodenkamper, L. J. Lembo, J. C. Brock, and M. C. Wu, "Characterization of a coherent optical RF channelizer based on a diffraction grating," IEEE T. Microw. Theory, vol. 49, no. 10, pp. 1996-2001, Oct. 2001.

[153] W. T. Rhodes, “Acousto-optic signal processing: Convolution and correlation," Proc. IEEE, vol. 69, no. 1, pp. 65-79, Jan. 1981.

[154] D. B. Hunter, L. G. Edvell, and M. A. Englund, "Wideband microwave photonic channelised receiver," Int. Topical Meeting on Microw. Photon., pp. 249-251, 2005-Oct.

[155] S. T. Winnall, A. C. Lindsay, M. W. Austin, J. Canning, and A. Mitchell, "A microwave channelizer and spectroscope based on an integrated optical Bragg-grating Fabry-Perot and integrated hybrid fresnel lens system," IEEE T. Microw. Theory, vol. 54, no. 2, pp. 868-872, Feb. 2006.

[156] W. Y. Xu, D. Zhu, and S. L. Pan, "Coherent photonic radio frequency channelization based on dual coherent optical frequency combs and stimulated Brillouin scattering," Opt. Eng., vol. 55, no. 4, Apr. 2016.

[157] X. H. Zou, W. Z. Li, W. Pan, L. S. Yan, and J. P. Yao, "Photonic-Assisted Microwave Channelizer With Improved Channel Characteristics Based on Spectrum-Controlled Stimulated Brillouin Scattering," IEEE T. Microw. Theory, vol. 61, no. 9, pp. 34703478, Sep. 2013.

[158] Choudhary, B. Morrison, I. Aryanfar, S. Shahnia, M. Pagani, Y. Liu, K. Vu, S. Madden, D. Marpaung, and B. J. Eggleton, "Advanced Integrated Microwave Signal Processing with Giant On-Chip Brillouin Gain,” J. Lightwave Technol., vol. 35, no. 4, 846854 (2017).

[159] S. Bres, S. Zlatanovic, A. O. J. Wiberg, J. R. Adleman, C. K. Huynh, E. W. Jacobs, J. M. Kvavle, and S. Radic, "Parametric Photonic Channelized RF Receiver," IEEE Photonic Tech. L., vol. 23, no. 6, pp. 344-346, Mar. 2011. 
[160] O. J. Wiberg, D. J. Esman, L. Liu, J. R. Adleman, S. Zlatanovic, V. Ataie, E. Myslivets, B. P. P. Kuo, N. Alic, E. W. Jacobs, and S. Radic, "Coherent Filterless Wideband Microwave/Millimeter-Wave Channelizer Based on Broadband Parametric Mixers," J. Lightwave Technol., vol. 32, no. 20, pp. 3609-3617, Oct. 2014.

[161] X. H. Zou, W. Pan, B. Luo, and L. S. Yan, "Photonic approach for multiple-frequency-component measurement using spectrally sliced incoherent source," Opt. Lett., vol. 35, no. 3, pp. 438-440, Feb. 2010.

[162]F.Volkening,"Photonic channelized RF receiver employing dense wavelength division multiplexing,”U.S.Patent 724 583 3B1, 2007.

[163] X. J. Xie, Y. T. Dai, K. Xu, J. Niu, R. X. Wang, L. Yan, and J. T. Lin, "Broadband Photonic RF Channelization Based on Coherent Optical Frequency Combs and I/Q Demodulators," IEEE Photonics J., vol. 4, no. 4, pp. 1196-1202, Aug. 2012.

[164] Z. Li, X. M. Zhang, H. Chi, S. L. Zheng, X. F. Jin, and J. P. Yao, "A Reconfigurable Microwave Photonic Channelized Receiver Based on Dense Wavelength Division Multiplexing Using an Optical Comb,” Opt. Commun., vol. 285, no. 9, pp. 2311-2315, 2012.

[165] R. Y. Li, H. W. Chen, Y. Yu, M. H. Chen, S. G. Yang, and S. Z. Xie, "Multiple-frequency measurement based on serial photonic channelization using optical wavelength scanning," Opt. Lett., vol. 38, no. 22, pp. 4781-4784, Nov. 2013.

[166] W. H. Hao, Y. T. Dai, F. F. Yin, Y. Zhou, J. Q. Li, J. Dai, W. Z. Li, and K. Xu, "Chirped-pulse-based broadband RF channelization implemented by a mode-locked laser and dispersion," Opt. Lett., vol. 42, no. 24, pp. 5234-5237, Dec. 2017.

[167] T. Herr, K. Hartinger, J. Riemensberger, C. Y. Wang, E. Gavartin, R. Holzwarth, M. L. Gorodetsky, and T. J. Kippenberg, "Universal formation dynamics and noise of Kerr-frequency combs in microresonators," Nat. Photonics, vol. 6, no. 7, 480-487 (2012).

[168] L. Caspani, C. Xiong, B. Eggleton, D. Bajoni, M. Liscidini, M. Galli, R. Morandotti, David J. Moss, "On-chip sources of quantum correlated and entangled photons", Nature: Light Science and Applications, vol. 6, e17100 (2017); doi: 10.1038/lsa.2017.100.

[169] F. Da Ros, E. P. da Silva, D. Zibar, S. T. Chu, B. E. Little, R. Morandotti, M. Galili, D. J. Moss, and L. K. Oxenlowe, "Wavelength conversion of QAM signals in a low loss CMOS compatible spiral waveguide," APL Photonics, vol. 2, no. 4 (2017).

[170] X. X. Xue and A. M. Weiner, "Microwave photonics connected with microresonator frequency combs," Front. Optoelectron., vol. 9, no. 2, pp. 238-248, Jun. 2016.

[171] S. Coen, H. G. Randle, T. Sylvestre, and M. Erkintalo, "Modeling of octave-spanning Kerr frequency combs using a generalized mean-field Lugiato-Lefever model," Opt. Lett., vol. 38, no. 1, pp. 37-39, Jan. 2013.

[172] Y. K. Chembo and C. R. Menyuk, "Spatiotemporal Lugiato-Lefever formalism for Kerr-comb generation in whispering-gallerymode resonators," Phys. Rev. A., vol. 87, no. 5, May. 2013.

[173] H. Guo, M. Karpov et al., "Universal dynamics and deterministic switching of dissipative Kerr solitons in optical microresonators," Nat. Phys., vol. 13, no. 1, pp. 94-102, Jan. 2017.

[174] X. Xue, Y. Xuan, C. Wang, P.-H. Wang, Y. Liu, B. Niu, D. E. Leaird, M. Qi, and A. M. Weiner, "Thermal tuning of Kerr frequency combs in silicon nitride microring resonators", Opt. Express, vol. 24, no. 1, pp. 687-698, Jan. 2016.

[175] B. Bernhardt, A. Ozawa, P. Jacquet, M. Jacquey, Y. Kobayashi, T. Udem, R. Holzwarth, G. Guelachvili, T. W. Hansch, and N. Picque, "Cavity-enhanced dual-comb spectroscopy," Nat. Photonics, vol. 4, no. 1, pp. 55-57, Jan. 2010

[176] T. Ideguchi, et al., "Adaptive real-time dual-comb spectroscopy”, Nat. Commun., vol. 5, 3375, 2014.

[177] G. Millot, S. Pitois, M. Yan, T. Hovhannisyan, A. Bendahmane, T. W. Hansch, and N. Picque, "Frequency-agile dual-comb spectroscopy," Nat. Photonics, vol. 10, no. 1, pp. 27-37, Jan. 2016.

[178] M. G. Suh, Q. F. Yang, K. Y. Yang, X. Yi, and K. J. Vahala, “Micro-resonator soliton dual-comb spectroscopy,” Science, vol. 354, no. 6312, pp. 600-603, Nov. 2016.

[179] N. G. Pavlov, G. Lihachev, S. Koptyaev, E. Lucas, M. Karpov, N. M. Kondratiev, I. A. Bilenko, T. J. Kippenberg, and M. L. Gorodetsky, "Soliton dual frequency combs in crystalline microresonators," Opt. Lett., vol. 42, no. 3, pp. 514-517, Feb. 2017.

[180] T. C. Briles, T. Drake, D. Spencer, J. R. Stone, C. Fredrick, Q. Li, D. Westly, B. R. Ilic, X. Yi, K. Y. Yang, K. Vahala, K. Srinivasan, S. Diddams, and S. Papp, "Optical Frequency Synthesis Using a Dual-Kerr-Microresonator Frequency Comb," in Conference on Lasers and Electro-Optics, OSA Technical Digest (online) (Optical Society of America, 2017), paper SW4N.3.

[181] B. E. Little, S. T. Chu, P. P. Absil, J. V. Hryniewicz, F. G. Johnson, F. Seiferth, D. Gill, V. Van, O. King, and M. Trakalo, "Very high-order microring resonator filters for WDM applications," IEEE Photonics Technology Letters, vol. 16, no. 10, 2263-2265. 2004.

[182] J. Wu, T. Moein, X. Xu, and D. J. Moss, "Advanced photonic filters based on cascaded Sagnac loop reflector resonators in siliconon-insulator nanowires," APL Photonics, vol. 3, 046102 (2018). DOI:/10.1063/1.5025833Apr. 2018.

[183] J. Wu, T. Moein, X. Xu, G. H. Ren, A. Mitchell, and D. J. Moss, "Micro-ring resonator quality factor enhancement via an integrated Fabry-Perot cavity,” APL Photonics, vol. 2, 056103 (2017).

[184] H. Arianfard, J. Wu, S. Juodkazis, and D. J. Moss, “Advanced Multi-Functional Integrated Photonic Filters Based on Coupled Sagnac Loop Reflectors", Journal of Lightwave Technology, Vol. 39, (5) pp.1400-1408 (2021). DOI: 10.1109/JLT.2020.3037559.

[185] Mengxi Tan, Xingyuan Xu, Jiayang Wu, Thach G. Nguyen, Sai T. Chu, Brent E. Little, Roberto Morandotti, Arnan Mitchell, and David J. Moss, "Photonic Radio Frequency Channelizers based on Kerr Micro-combs and Integrated Micro-ring Resonators", JOSarXiv.202010.0002.

[186] Mengxi Tan, Xingyuan Xu, Jiayang Wu, Thach G. Nguyen, Sai T. Chu, Brent E. Littl, Arnan Mitchell, Roberto Morandotti, and David J. Moss, "Orthogonally polarized RF optical single sideband generation with integrated ring resonators", JOSarXiv.202010.0001.

[187] M. Tan, X. Xu, J. Wu, T. G. Nguyen, Sai T. Chu, B. E. Littl, A. Mitchell,R. Morandotti, and D. J. Moss, "Orthogonally polarized RF optical single sideband generationwith integrated ring resonators", In Press, Journal of Semiconductors (2021). http://www.jos.ac.cn/article/shaid/4c314db6c6dcfe19c275efde2b99a22329ba658eda52233b8ab4b8a08b55a7bf.

[188] M Sinobad et al., Optica, Vol. 5 (4), 360-366 (2018). 